\title{
Type-II Calabi-Yau compactifications, T-duality and special geometry in general spacetime signature
}

\author{
M. Médevielle, T. Mohaupt and G. Pope \\ Department of Mathematical Sciences, University of Liverpool, \\ Peach Street, Liverpool L69 7ZL, U.K. \\ E-mail: maxime.medevielle@liverpool.ac.uk, Thomas.Mohaupt@liv.ac.uk, \\ giacomo@liverpool.ac.uk
}

Abstract: We obtain the bosonic Lagrangians of vector and hypermultiplets coupled to four-dimensional $\mathcal{N}=2$ supergravity in signatures $(0,4),(1,3)$ and $(2,2)$ by compactification of type-II string theories in signatures $(0,10),(1,9)$ and $(2,8)$ on a Calabi-Yau threefold. Depending on the signature and the distinctions between type-IIA/IIA*/IIB/IIB*/IIB' the resulting scalar geometries are special Kähler or special para-Kähler for vector multiplets and quaternion-Kähler or para-quaternion Kähler for hypermultiplets. By spacelike and timelike reductions we obtain three-dimensional $\mathcal{N}=4$ supergravity theories coupled to two sets of hypermultiplets. We determine the c-maps relating vector to hypermultiplets, and show how the four-dimensional theories are related by spacelike, timelike and mixed, signature-changing T-dualities.

KEYwords: String Duality, Supergravity Models, Superstrings and Heterotic Strings

ARXIV EPRINT: 2111.09017 


\section{Contents}

1 Introduction 1

2 Vector and hypermultiplets in four and three dimensions 4

2.1 Supersymmetry algebras in four and three dimensions 4

2.2 Vector multiplets 5

2.3 Hypermultiplets $\quad 7$

2.4 Reduction to three dimensions 9

3 Ten-dimensonal type-II string theories $\quad 11$

4 Type-II Calabi-Yau compactifications $\quad 14$

$\begin{array}{lll}4.1 & \text { Type-IIA Calabi-Yau compactifications } & 15\end{array}$

$\begin{array}{lll}4.1 .1 & \text { The vector multiplet sector } & 17\end{array}$

$\begin{array}{ll}\text { 4.1.2 The hypermultiplet sector } & 18\end{array}$

$\begin{array}{ll}4.2 \text { Type-IIB Calabi-Yau compactifications } & 19\end{array}$

5 T-duality $\quad \mathbf{2 1}$

5.1 Signature $(1,3)$ and spacelike/timelike T-duality 22

$\begin{array}{lll}5.2 & \text { Mixed T-dualities and signature change } & 23\end{array}$

6 Outlook 26

$\begin{array}{lr}\text { A Calabi-Yau compactifications } & 27\end{array}$

$\begin{array}{ll}\text { A.1 Calabi-Yau threefolds } & 27\end{array}$

A.2 Ten-dimensional Lagrangians 32

A.3 Reduction of the graviton-dilaton sector 33

A.4 Contribution of the $B$-field to the vector multiplet sector 33

A.5 Contribution of R-R-kinetic terms to the vector multiplet sector 34

A.6 Contribution of the topological terms to the vector multiplet sector $\quad 35$

A.7 Final result for the gravity and vector multiplet sector 35

A.8 Contribution of the kinetic R-R-terms to the hypermultiplet sector 36

A.9 Contribution of topological terms and of the $B$-field to the hypermultiplet sector $\quad 37$

$\begin{array}{ll}\text { A.10 Final result for the hypermultiplet sector } & 37\end{array}$ 


\section{Introduction}

String theory is a web of perturbatively defined theories which are related to each other by various dualities. In particular, ten-dimensional type-II string theories, which have the maximal amount of supersymmetry, are related to each other by T-duality and S-duality. If one includes timelike T-duality, then besides the familiar type-IIA and type-IIB theories there exist two further theories in Lorentz signature, type-IIA* and type-IIB*, and there also exist further type-II theories in all possible ten-dimensional spacetime signatures [1-3]. The formal properties of these theories as well as their potential applications in model building and cosmology have been investigated further in $[4,5]$. Exotic type-II theories have unusual features and their ultimate role in string theory remains to be understood. From the point of view of symmetries and string geometry, it is natural to include them. Timelike dimensional reduction is a valid solution-generating technique, and timelike T-dualities exist whenever one can find an alternative dimensional up-lift. Symmetries which become manifest in dimensional reduction give information about the hidden symmetries of the full theory [6]. Including time in the reduction as a strategy for uncovering the full symmetry structure underlying string theory has been advocated in [7]. In the frameworks of doubled and exceptional geometry and field theory, type-II* theories seem to be on the same footing as the conventional ones $[8,9]$. Since type-II and type-II* theories have the same Euclideanized version [1], it is natural to think of them as resulting from the same underlying Euclidean partition function.

When combining timelike T-duality with S-duality, string symmetries also lead to backgrounds with non-Lorentzian signatures. While their interpretation is challenging, they cannot be discarded ad hoc, since string theory is believed to be a single theory with all of its consistent backgrounds connected by physical processes. The relevant question is therefore whether vacua with exotic signature can be generated, and evidence for this has been presented in [4]. It has also been argued that string theories in exotic signature can be defined holographically as duals of gauge theories based on Lie supergroups [4]. We also note that the network of type-II string theories and of the related eleven-dimensional M-theories realizes all possible ten- and eleven-dimensional supersymmetry algebras with 32 real supercharges [10], so that when allowing exotic signatures in string theory, all maximally symmetric supergravities in all signatures can be realized as limits. Finally, the inclusion of non-Lorentzian signatures is natural from the point of view of the Euclidean approach to quantum gravity, since complex saddle points contribute to the functional integral. Recently, the role of complex spacetime metrics in quantum field theory and quantum gravity has been emphasised in [11-13]. As a natural extension, one can complexify all fields, which would imply to consider all type-II theories as part of a single complex configuration space. We remark that complex saddle points can contribute to Euclidean path integrals for scalar fields, and that there are examples where actions with inverted kinetic terms can be viewed as arising from manipulating integration contours in complexified field space, see [14] for an elementary example.

Calabi-Yau compactifications of Lorentz signature type-IIA/B string theories give rise to four-dimensional $\mathcal{N}=2$ supergravity theories with vector and hypermultiplets [15-17]. 
This is a much studied class of theories which while not phenomenologically realistic, has rich and complex dynamics, since the scalar geometry is not rigid but depends on functions of the scalar fields. $\mathcal{N}=2$ supersymmetry still severely restricts the quantum and stringy corrections that these functions can receive, so that one often can find exact non-perturbative results. Applications range from the study of field theories, to black holes and their entropy, and to the AdS/CFT correspondence. It is therefore interesting to extend these studies to the Calabi-Yau compactifications of exotic type-II theories.

The Calabi-Yau compactification of the type-IIA theory in Euclidean signature $(0,10)$ has been worked out in [18]. Moreover, the vector multiplet sectors of five- and fourdimensional supergravity in arbitrary signature have been found in [19] through an analysis of Killing spinor equations combined with the reductions of eleven-dimensional supergravity theories in signatures $(1,10),(2,9)$ and $(5,6)$ on Calabi-Yau threefolds, followed by the reduction to four dimensions on spacelike and timelike circles.

In this paper we will obtain the bosonic actions for four-dimensional $\mathcal{N}=2$ supergravity coupled to vector and hypermultiplets in signatures $(0,4),(1,3)$ and $(2,2)$ by compactification of type-II string theories in signatures $(0,10),(1,9),(2,8)$ on Calabi-Yaur threefolds. Carrying out these reductions is straightforward since for type-IIA one can adapt the computations of $[16,18]$, while the corresponding results for type-IIB are fixed by mirror symmetry. Therefore, our main focus is the interpretation of the relative sign flips between terms in the resulting four-dimensional Lagrangians in terms of the special geometry of their vector and hypermultiplet manifolds. These geometries vary between signatures and between type-II and type-II*. There is an intimate relation between these signs and the variation of the R-symmetry groups of the underlying supersymmetry algebras between signatures, and between standard and twisted (type-*) supersymmetry algebras.

As is well known, in signature $(1,3)$ the scalar geometry of vector multiplets coupled to supergravity is special Kähler, while the geometry of hypermultiplets is quaternionic-Kähler, see [20-23] for review. In Euclidean signature the scalar geometry of vector multiplets becomes special para-Kähler, which is reflected by a change of the abelian factor of the R-symmetry group from $\mathrm{U}(1)$ to $\mathrm{SO}(1,1)[24,25]$. In three Euclidean dimensions the geometry of hypermultiplets is para-quaternionic Kähler [26]. In [27] it was observed that there exists a twisted version of the $\mathcal{N}=2$ supersymmetry algebra, which has a non-compact R-symmetry group. The vector multiplet Lagrangian differs from the standard one by a sign flip of some kinetic terms, which is analogous to the difference between type-II and type-II* theories. The same type of sign flip was observed in [19], when reducing five-dimensional vector multiplets coupled to supergravity from signature $(2,3)$ to signature $(1,3)$. One should therefore expect that $\mathcal{N}=2$ theories which realize the twisted $\mathcal{N}=2$ algebra can be obtained as Calabi-Yau compactifications of type-II* theories. In this paper we will verify this explicitly, as part of obtaining a complete list of scalar geometries for four-dimensional $\mathcal{N}=2$ supergravity with vector and hypermultiplets for all signatures through the dimensional reduction of type-II theories on Calabi-Yau threefolds.

In addition, we will perform all possible spacelike and timelike dimensional reductions from four to three dimensions. After reduction, vector multiplets can be dualized into hypermultiplets, so that one obtains a scalar manifold which is the product of two hyper- 
multiplet manifolds. The map relating a vector multiplet manifold to a hypermultiplet by reduction is know as the c-map [28, 29]. By starting in arbitrary signature and including timelike reductions, one obtains variants of the c-map, which we describe for all possible cases. Whenever a dimensional reduction can be combined with a different dimensional lifting (equivalently, whenever the same three-dimensional theory can be obtained from two different four-dimensional theories by reduction), this realizes a T-duality between the underlying type-II string theories. We map out the complete network of spacelike, timelike and mixed T-dualities, where mixed T-dualities combine spacelike reduction/lifting with timelike lifting/reduction and thus change the four-dimensional signature.

We briefly mention further motivations and future application of our work. One is the study of solutions to four-dimensional $\mathcal{N}=2$ theories with twisted supersymmetry and with non-Lorentzian signature, as well as their dimensional uplifts to ten and eleven dimensions. In particular, according to [30, 31], there is a correspondence between the planar cosmological solutions in $\mathcal{N}=2$ vector multiplet theories that can be embedded into type-II string theory, and planar black hole solutions in vector multiplet theories realizing the twisted $\mathcal{N}=2$ supersymmetry algebra, which, as we show in this paper, can be embedded into type-II*. Both solutions can be related to the same four-dimensional Euclidean partition function, which explains that their Killing horizons satisfy the same thermodynamic relations [31]. This is consistent with type-II and type-II* having the same Euclideanized form [1]. For other work on solutions of exotic $\mathcal{N}=2$ theories see [32-37].

Another potential application is topological string theory. Standard Type-II Calabi-Yau compactifications allow two topological twists, which define two topological worldsheet theories, the A-model and the B-model, which are sensitive to the Kähler and complex structure moduli respectively. Since Calabi-Yau compactifications of exotic type-II theories work analogously to standard type-II theories, and given that we will show that the geometry of the resulting moduli spaces can be determined in the supergravity approximation, we expect that a world-sheet perspective for these compactifications can be developed too. Topological string theories encode a subsector of the full string theories, and may also be related to a 'topological phase' of string theory, where more of its symmetries become manifest [38]. We remark that in such a topological phase, the expectation value of the spacetime metric is zero, which makes it natural that phases with non-Lorentzian signature coexist in the theory with conventional Lorentzian phases.

The outline of this paper is as follows. We start from the classification of fourdimensional $\mathcal{N}=2$ and three-dimensional $\mathcal{N}=4$ supersymmetry algebras and explain how most of the qualitative features of the scalar geometries of vector and hypermultiplets as well as their mutual relations by T-dualities can already be predicted by inspection of the R-symmetry groups. We present the bosonic vector and hypermultiplet Lagrangians, and explain the effects of changing the supersymmetry algebra on the scalar geometries. This includes a brief review of special para-Kähler and para-quaternion-Kähler geometries, which replace the familar special Kähler and quaternion-Kähler geometries for certain signatures. We perform all possible spacelike and timelike reductions from signatures $(0,4),(1,3)$ and $(2,2)$ to signatures $(0,3)$ and $(1,2)$, and show that the six resulting c-maps which map vector multiplet manifolds to hypermultiplet manifolds fall into three distinct 
classes, depending on whether the resulting hypermultiplet manifold is quaternionic-Kähler, para-quaternionic-Kähler with a special Kähler base or para-quaternionic-Kähler with a special para-Kähler base.

Then we review type-II string theories in ten dimensions and catalogue the relative sign flips of their kinetic terms. Next, we explain how these sign flips affect compactifications on Calabi-Yau threefolds, and obtain the corresponding sign flips of the resulting fourdimensional vector and hypermultiplet Lagrangians. While in the main part of the paper we just trace the kinetic terms, we provide a full derivation in the appendix. Here we use that the reduction of all individual terms is available from the work of [16] on the reduction of type-IIA with signature $(1,9)$ and of $[18]$ on the reduction of type-IIA with signature $(0,10)$. Combining the results from dimensional reduction with the previous results on c-maps we obtain six types of T-dualities by identifying all possible combinations of reductions from four to three with 'oxidations' from three to four dimensions. These T-dualities organise into two orbits, one which relates type-IIA/IIB/IIA*/IIB* through 'pure' - that is spatial or timelike T-dualities, the other which relates type-IIA ${ }_{(0,10)} / \mathrm{IIB}_{(0,9)} / \mathrm{IIA}_{(2,8)}$ through 'mixed', signature changing T-dualities. This separation coincides with the one between worldsheet theories with Lorentzian and with Euclidean signature [2]. Both orbits could only be related through the S-duality between type-IIB* and type-IIB', which is not expected to be valid for generic $\mathcal{N}=2$ compactifications, though it may be realized for non-generic ' $\mathcal{N}=4$-like' compactifications.

\section{Vector and hypermultiplets in four and three dimensions}

\subsection{Supersymmetry algebras in four and three dimensions}

Four-dimensional $\mathcal{N}=2$ supersymmetry algebras, that is four-dimensional supersymmetry algebras with eight real supercharges, ${ }^{1}$ have been classified for arbitrary signature in [27]. They are completely characterized by their R-symmetry groups, which we list in table 1. While the $\mathcal{N}=2$ algebra is unique in Euclidean signature $(0,4)$ and in neutral signature $(2,2)$, there are two non-isomorphic algebras in Lorentz signature $(1,3) .^{2}$ Besides the standard $\mathcal{N}=2$ algebra with compact R-symmetry group $\mathrm{U}(2)$ there exists a second algebra with non-compact R-symmetry $\mathrm{U}(1,1)$, which we will refer to as the twisted $\mathcal{N}=2$ algebra. The change of the R-symmetry group reflects itself in certain sign flips in the bosonic Lagrangian [27], which are similar to those which distinguish type-II and type-II* string theories [1]. We will see later that theories realizing the twisted $\mathcal{N}=2$ algebra are obtained by the compactification of type-II* string theories on Calabi-Yau threefolds. The uniqueness of the supersymmetry algebras in Euclidean and neutral signature reflects the uniqueness

\footnotetext{
${ }^{1}$ In Euclidean signature this is the smallest supersymmetry algebra. Our convention is to count supersymmetries in multiples of Majorana spinors, irrespective of whether Majorana spinor spinors exist is the particular signature. This convention is natural if one considers supersymmetry algebras in different signatures at the same time.

${ }^{2}$ We use the mostly plus convention, so $(1,3)$ means that the metric has 3 positive eigenvalues and 1 negative eigenvalue.
} 


\begin{tabular}{|l|l|l|l|}
\hline Signature & R-symmetry & VM geometry & HM geometry \\
\hline$(0,4)$ & $\mathrm{U}(2)^{*} \cong \mathrm{SO}(1,1) \times \mathrm{SU}(2)$ & SPK & QK \\
$(1,3)$ & $\mathrm{U}(2) \cong \mathrm{U}(1) \times \mathrm{SU}(2)$ & $\mathrm{SK}_{+}$ & QK \\
& $\mathrm{U}(1,1) \cong \mathrm{U}(1) \times \mathrm{SU}(1,1)$ & $\mathrm{SK}_{-}$ & PQK \\
$(2,2)$ & $\mathrm{GL}(2, \mathbb{R}) \cong \mathrm{SO}(1,1) \times S L^{ \pm}(2, \mathbb{R})$ & SPK & PQK \\
\hline
\end{tabular}

Table 1. Four-dimensional $\mathcal{N}=2$ supersymmetry algebras, their R-symmetry groups and their scalar geometries. We use the acronyms SK = special Kähler, SPK = special para-Kähler, QK = quaternionic Kähler and PQK = para-quaternionic Kähler. See section 2.2 for further explanations.

\begin{tabular}{|l|l|l|l|}
\hline Signature & R-symmetry & $\mathrm{HM}_{1}$ geometry & $\mathrm{HM}_{2}$ geometry \\
\hline$(0,3)$ & $\mathrm{SO}^{*}(4) \cong \mathrm{SL}(2, \mathbb{R}) \times \mathrm{SU}(2)$ & $\mathrm{PQK}$ & $\mathrm{QK}$ \\
$(1,2)$ & $O(4) \cong \mathrm{SU}(2) \times \mathrm{SU}(2)$ & $\mathrm{QK}$ & $\mathrm{QK}$ \\
& $O(1,3)$ & - & - \\
& $O(2,2) \cong \mathrm{SL}(2, \mathbb{R}) \times \mathrm{SL}(2, \mathbb{R})$ & $\mathrm{PQK}$ & $\mathrm{PQK}$ \\
\hline
\end{tabular}

Table 2. Three-dimensional $\mathcal{N}=4$ supersymmetry algebras, their R-symmetry groups and their scalar geometries. See section 2.3 for further explanations.

\begin{tabular}{|l|l|l|l|l|l|l|}
\hline Signature & R-symmetry & Geometry & Reduction & R-symmetry & Geometry & c-map \\
\hline$(0,4)$ & $\mathrm{SO}(1,1) \times \mathrm{SU}(2)$ & $\mathrm{SPK} \times \mathrm{QK}$ & $(0,4) \rightarrow(0,3)$ & $\mathrm{SL}(2, \mathbb{R}) \times \mathrm{SU}(2)$ & $\mathrm{PQK} \times \mathrm{QK}$ & Euclidean c-map \\
\hline \multirow{2}{*}{$(1,3)$} & $\mathrm{U}(1) \times \mathrm{SU}(2)$ & $\mathrm{SK} \times \mathrm{QK}$ & $(1,3) \rightarrow(0,3)$ & $\mathrm{SL}(2, \mathbb{R}) \times \mathrm{SU}(2)$ & $\mathrm{PQK} \times \mathrm{QK}$ & Temporal c-map \\
& $\mathrm{U}(1) \times \mathrm{SU}(1,1)$ & $\mathrm{SK} \times \mathrm{QK}$ & $(1,3) \rightarrow(0,3)$ & $\mathrm{SU}(2) \times \mathrm{SU}(1,1)$ & $\mathrm{QK} \times \mathrm{PQK}$ & Twisted temporal c-map \\
\cline { 5 - 7 } & $\mathrm{U}(1) \times \mathrm{SU}(2)$ & $\mathrm{SK} \times \mathrm{QK}$ & $(1,3) \rightarrow(1,2)$ & $\mathrm{SU}(2) \times \mathrm{SU}(2)$ & $\mathrm{QK} \times \mathrm{QK}$ & $($ spatial $)$ c-map \\
& $\mathrm{U}(1) \times \mathrm{SU}(1,1)$ & $\mathrm{SK} \times \mathrm{PQK}$ & $(1,3) \rightarrow(1,2)$ & $\mathrm{SU}(1,1) \times \mathrm{SU}(1,1)$ & $\mathrm{PQK} \times \mathrm{PQK}$ & Twisted (spatial) c-map \\
& $\mathrm{SO}(1,1) \times S L^{ \pm}(2, \mathbb{R})$ & $\mathrm{SPK} \times \mathrm{PQK}$ & $(2,2) \rightarrow(1,2)$ & $\mathrm{SU}(1,1) \times \mathrm{SU}(1,1)$ & $\mathrm{PQK} \times \mathrm{PQK}$ & Neutral c-map \\
\hline$(2,2)$ & &
\end{tabular}

Table 3. Dimensional reduction from four to three dimensions for all inequivalent signatures: R-symmetry groups, scalar geometries, and type of c-map.

of type-II string theories in signatures $(0,10)$ and $(2,8)$, from which such theories can again be obtained as Calabi-Yau compactifications.

Three-dimensional $\mathcal{N}=4$ supersymmetry algebras have been classified, for arbitrary signature in [10], and are again characterized uniquely by their R-symmetry groups, see table 2. The embeddings $\mathrm{U}^{*}(2) \subset \mathrm{SO}^{*}(4), \mathrm{U}(2) \subset O(4), \mathrm{U}(1,1) \subset O(2,2)$ and $\mathrm{GL}(2, \mathbb{R}) \subset$ $O(2,2)$ indicate how these algebras are related to four-dimensional $\mathcal{N}=2$ algebras by spacelike or timelike dimensional reduction, see table 3 . There is no candidate for a dimensional lift of the algebra with R-symmetry $O(1,3)$. In the following sections we will review vector and hypermultiplets, in particular, the geometry of their scalar manifolds, and how this geometry is tied to the R-symmetry group.

\subsection{Vector multiplets}

We start in signature $(1,3)$ with the standard $\mathcal{N}=2$ supersymmetry algebra with Rsymmetry group $\mathrm{U}(2) \cong \mathrm{U}(1) \times \mathrm{SU}(2)$. A vector multiplet contains a complex scalar $z$, an 
$\mathrm{SU}(2)$ doublet of spinors, and a gauge field $\mathcal{A}_{\mu}$. The scalar and gauge field are neutral under $\mathrm{SU}(2)$. Under the $\mathrm{U}(1)$, the scalars, spinors and vectors carry charges $\mp 1, \mp \frac{1}{2}, 0$ respectively. The scalar manifold is an affine special Kähler manifold for rigid supersymmetry and a projective special Kähler manifold for local supersymmetry. We refer to [22] for a review of special geometry which uses the same conventions and terminology as used in this paper. Both types of special Kähler geometries (SK geometries) have in common that the Kähler metric $g_{\alpha \bar{\beta}}(z, \bar{z}), \alpha, \beta=1, \ldots, n_{V}$ of the scalar manifold can be expressed in terms of a holomorphic function $\mathcal{F}\left(z^{\alpha}\right)$, called the prepotential. Special Kähler geometry is intimately related to the invariance of the field equations under symplectic transformations, which generalize and contain electric-magnetic duality transformations [39, 40]. We are interested in the case where the $n_{V}$ vector multiplets are coupled to $\mathcal{N}=2$ supergravity. The supergravity multiplet contains one further vector field $\mathcal{A}_{\mu}^{0}$. A simple, linear action of the symplectic group $\operatorname{Sp}\left(2 n_{V}+2, \mathbb{R}\right)$ is obtained by taking certain field-dependent linear combinations $A_{\mu}^{I}, I=0,1, \ldots, n_{V}$ of the vector fields $\mathcal{A}_{\mu}^{0}, A_{\mu}^{\alpha}$. The associated field strengths $F_{\mu \nu}^{I}$, when combined with their duals $G_{I \mid \mu \nu}$ form a vector $\left(F_{\mu \nu}^{I}, G_{I \mid \mu \nu}\right)$ which transforms linearly under $\operatorname{Sp}\left(2 n_{V}+2, \mathbb{R}\right)$. The dual field strengths are dependent quantities, which are defined as $G_{I \mid \mu \nu}^{ \pm}=\partial \mathcal{L} / \partial F_{\mu \nu}^{ \pm \mid I}$, where $\mathcal{L}$ is the Lagrangian, and where $F_{\mu \nu}^{ \pm \mid I}$ and $G_{I \mid \mu \nu}^{ \pm}$ are the (anti-)selfdual parts of $F_{\mu \nu}^{I}$ and $G_{I \mid \mu \nu}$. We remark that the linear action of the symplectic group is obvious if one uses the gauge equivalence between $\mathcal{N}=2$ Poincaré supergravity with $n_{V}$ vector and $n_{H}$ hypermultiplets to $\mathcal{N}=2$ conformal supergravity with $n_{V}+1$ vector and $n_{H}+1$ hypermultiplets. In the superconformal setting $A_{\mu}^{I}$ are the vector fields of the $n_{V}+1$ superconformal vector multiplets. The corresponding scalars $X^{I}$ allow a symplectically covariant description of the scalar sector. In terms of the $X^{I}$ the prepotential is a holomorphic function $F(X)$ which is homogeneous of degree $2, F(\lambda X)=\lambda^{2} F(X)$. Combining the scalars $X^{I}$ with $F_{I}=\partial F / \partial X^{I}$ one obtains another symplectic vector $\left(X^{I}, F_{I}\right)$. The scalars $z^{\alpha}$ can be recovered as ratios $z^{\alpha}=X^{\alpha} / X^{0}$. The couplings between scalar and vector fields are encoded in a complex matrix $\mathcal{N}_{I J}=\mathcal{R}_{I J}+i \mathcal{I}_{I J}$, which can be expressed in terms of the prepotential. The kinetic terms for the scalar and vector fields are positive definite if $g_{\alpha \bar{\beta}}$ is positive definite and if $\mathcal{I}_{I J}$ is negative definite (in our convention).

The Lagrangian for the bosonic degrees of the supergravity multiplet and of $n_{V}$ vector multiplets takes the form

$$
L_{G+\mathrm{VM}}=\frac{1}{2} \star R_{4}-g_{\alpha \bar{\beta}}(z, \bar{z}) d z^{\alpha} \wedge \star d \bar{z}^{\bar{\beta}}-\frac{\lambda}{4} \mathcal{I}_{I J} F^{I} \wedge \star F^{J}+\frac{1}{4} \mathcal{R}_{I J} F^{I} \wedge F^{J},
$$

where $\lambda=-1$.

We now turn to the modifications which occur if we change the supersymmetry algebra. In signature $(1,3)$ we have the twisted algebra with R-symmetry group $\mathrm{U}(1,1)$. For this algebra the Lagrangian takes exactly the same form, but with $\lambda=1$, that is, the signs of the kinetic terms for all vector fields are flipped [27]. ${ }^{3}$ While the scalar manifold remains the same (for a given prepotential), we will use the notation $\mathrm{SK}_{ \pm}=\mathrm{SK}_{\mp \lambda}$ to keep track of

\footnotetext{
${ }^{3}$ This sign flip had already been observed in [19] by comparing the reductions of vector multiplets coupled to supergravity from signatures $(1,4)$ and $(2,3)$ to signature $(1,3)$. See [27] for a detailed explanation how this sign flip is related to the underlying R-symmetry groups.
} 
the relative sign between scalar and vector fields. Note that $\mathrm{SK}_{+}$corresponds to the case with standard kinetic terms, $\lambda=-1$.

Something more drastic happens in signatures $(0,4)$ and $(2,2)$, where special Kähler geometry is replaced by special para-Kähler geometry. We will provide a concise summary and refer to the review [22] as well as the original papers [24, 25, 27] for details. Paracomplex geometries are modelled on the para-complex numbers (also called split complex numbers) in the same way as complex geometries are modelled on the complex numbers. The para-complex numbers are obtained by replacing the complex unit $i$, which satisfies $i^{2}=-1$ and $\bar{i}=-i$ by the para-complex unit $e$, which satisfies $e^{2}=1$ and $\bar{e}=-e$. This allows one to define 'para-analogues' of almost complex, complex, Hermitian, Kähler and of affine and projective special Kähler geometry. For example, an almost para-complex structure $J$ on an even-dimensional real manifold $\mathcal{M}$ is an endomorphism field $J \in \operatorname{End}(T \mathcal{M})$ which satisfies $J^{2}=\operatorname{Id}_{T \mathcal{M}}$ and has an equal number of eigenvalues \pm 1 . If $J$ is integrable, $\mathcal{M}$ admits local para-complex coordinates $z^{i}=x^{i}+e y^{i}$, and is a para-complex manifold. Special para-Kähler (SPK) geometry is the para-analogue of special Kähler geometry. All usual formulae take the same form (assuming some care in placing factors $e$ ), with the prepotential now a para-holomorphic function of para-complex scalar fields $z^{\alpha}$.

The change from complex to para-complex target geometry is reflected by the change in the abelian factor of the R-symmetry group. For special Kähler targets, the infinitesimal action of $\mathrm{U}(1) \subset \mathrm{U}(2)$ is given by multiplication by the complex structure $I$. Similarly the infinitesimal action of $\mathrm{SO}(1,1) \subset \mathrm{U}(1,1)$ is given by multiplication by the para-complex structure $J$ [24]. Thus table 1 tells us immediately that the vector multiplet geometry is SK for signature $(1,3)$ but SPK for signature $(0,4)$ and $(2,2)$. This can also be verified by explicit construction of the vector multiplet representations, which in addition fixes the relative sign between the scalar and vector field terms [24, 27]. As we will review later, SPK geometry arises when reducing Euclidean IIA supergravity on a Calabi-Yau threefold [18]. Note that if the scalar manifold is SPK, this relative sign does not really matter, that is we can take $\lambda=-1$ or $\lambda=1$, because this sign can be flipped by a local field redefinition [27]. This reflects that in signatures $(0,4)$ and $(2,2)$ the supersymmetry algebra is unique, whereas in signature $(1,3)$ there are two inequivalent supersymmetry algebras, whose vector multiplet representations are distinguished by the relative sign between scalar and vector field terms. In Minkowski signature sign flips of the gauge kinetic term map solutions of one theory to solutions of the other. For planar Reissner-Nordstrom-like solutions, this defines a map which exchanges the regions inside and outside horizons, and maps cosmological to black hole solutions [31]. In contrast, in Euclidean and neutral signature solutions with flipped vector kinetic terms are related to one another by a field redefinition [37].

\section{$2.3 \quad$ Hypermultiplets}

Hypermultiplets exist in all dimensions $D \leq 6$. Their field content is four real scalars and a doublet of spinors. The scalar geometry does not change under dimensional reduction. In Lorentz signature the scalar geometry is hyper-Kähler (HK) in the rigid case and quaternionKähler (QK) in the local case. A detailed review in conventions close to ours can be found in [21]. In both cases the scalar manifold $\mathcal{N}$ carries the action of a quaternionic structure, 
which is spanned (at least locally) by three complex structures $I_{i}, i=1,2,3$, which satisfy the quaternionic algebra, that is they mutually anticommute and satisfy $I_{i} I_{j}=I_{k}$ for $i, j, k$ cyclic. Hypermultiplet scalars are charged under a non-abelian subgroup SU(2) of the R-symmetry group, and the infinitesimal action of $\mathrm{SU}(2)$ is given by multiplication with the complex structures $I_{i}$. The corresponding finite action is given by the unit quaternions, $a 1+b I_{1}+c I_{2}+d I_{3}$, where $a^{2}+b^{2}+c^{2}+d^{2}=1$, which form a group isomorphic to $\mathrm{SU}(2)$. Three-dimensonal hypermultiplets can be obtained from four-dimensional vector multiplets by dimensional reduction. This induces a map between (generic) SK manifolds and (non-generic) QK manifolds. This map is known as the c-map [28, 29]. The resulting QK manifolds contain the SK manifold they are constructed from as a totally geodesic submanifold, and the QK manifold is a group bundle over an SK base.

Table 3 shows that in various four- and three-dimensional signatures a factor $\mathrm{SU}(2)$ of the R-symmetry group is replaced by $\mathrm{SU}(1,1)$ relative to the standard Lorentz signature algebra. This indicates that the quaternionic structure of the HM scalar manifold is replaced by a para-quaternionic structure. The para-quaternions (also called split quaternions) are obtained by replacing two of the three complex units by para-complex units. The paraquaternionic algebra is isomorphic to the algebra $\mathbb{R}(2)$ of real $2 \times 2$ matrices, and the group of unit para-quaternions is isomorphic to $\mathrm{SU}(1,1)$. The para-analogues of hyper-Kähler (HK) and quaternion-Kähler (QK) geometry are called para-hyper-Kähler (PHK) and para-quaternion-Kähler (PQK) geometry. We refer to [26, 41] and the review [22] for details. As we will discuss below, there are versions of the c-map which map SK and SPK manifolds to PQK manifolds.

One case where we expect that the hypermultiplet geometry is PQK is signature $(0,3)$. This has been verified explicitly by dimensional reduction from signature $(1,3)$ to signature $(0,3)$, which defines the temporal c-map, and form signature $(0,4)$ to signature $(0,3)$, which defines the Euclidean c-map [26]. More generally the results of [26] imply the following: suppose that $\mathcal{M}_{2 n_{V}}$ is a (projective) SK or SPK manifold with coordinates $z^{\alpha}=x^{\alpha}+i_{\epsilon_{1}} y^{\alpha}$, metric $g_{\alpha \bar{\beta}}, \alpha, \beta=1, \ldots, n_{V}$ and vector coupling matrix $\mathcal{N}_{I J}=\mathcal{R}_{I J}+i_{\epsilon_{1}} \mathcal{I}_{I J}$, where $\epsilon_{1}=-1$, $i_{-1}=i$ for SK and $\epsilon_{1}=1, i_{1}=e$ for SPK. In the SK case we assume that $g_{\alpha \bar{\beta}}$ is positive definite and that $\mathcal{I}_{I J}$ is negative definite. ${ }^{4}$ Consider the two-parameter family of bosonic Lagrangians for $n_{H}=n_{V}+1$ hypermultiplets,

$$
\begin{aligned}
L_{\mathrm{HM}}^{\left(\epsilon_{1}, \epsilon_{2}\right)}= & -g_{\alpha \bar{\beta}} d z^{\alpha} \wedge \star d \bar{z}^{\bar{\beta}}-\frac{1}{4} d \varphi \wedge \star d \varphi \\
& +\epsilon_{1} e^{-2 \varphi}\left[d \tilde{\phi}+\frac{1}{2}\left(\zeta^{I} d \tilde{\zeta}_{I}-\tilde{\zeta}_{I} d \zeta^{I}\right)\right] \wedge \star\left[d \tilde{\phi}+\frac{1}{2}\left(\zeta^{I} d \tilde{\zeta}_{I}-\tilde{\zeta}_{I} d \zeta^{I}\right)\right] \\
& -\frac{\epsilon_{2}}{2} e^{-\varphi}\left[\mathcal{I}_{I J} d \zeta^{I} \wedge \star d \zeta^{J}-\epsilon_{1} \mathcal{I}^{I J}\left(d \tilde{\zeta}_{I}+\mathcal{R}_{I K} d \zeta^{K}\right) \wedge \star\left(d \tilde{\zeta}_{I}+\mathcal{R}_{I K} d \zeta^{K}\right)\right],
\end{aligned}
$$

where $\epsilon_{2}= \pm 1$ is a second parameter. It was shown in [26], that the resulting HM manifold $\mathcal{N}_{4 n_{H}}=\mathcal{N}_{4 n_{V}+4}$ is QK for $\left(\epsilon_{1}=-1, \epsilon_{2}=-1\right)$ and PQK for the other three cases. Moreover for $\left(\epsilon_{1}=-1, \epsilon_{2}=1\right)$ the PQK manifold is a group bundle over an SK base (the space parametrized by the complex scalars $\left.z^{\alpha}\right)$, while for $\left(\epsilon_{1}=1, \epsilon_{2}= \pm 1\right)$ it is a group bundle over

\footnotetext{
${ }^{4}$ If this condition is relaxed one obtains QK manifolds of indefinite signature. See [42] for special geometry with indefinite signature SK and QK target spaces.
} 


\begin{tabular}{|l|l|l|l|}
\hline Domain & Image & Parameters & c-map \\
\hline SK & QK & $\epsilon_{1}=-1, \epsilon_{2}=-1$ & spatial \\
SK & PQK $_{\mathrm{SK}}$ & $\epsilon_{1}=-1, \epsilon_{2}=1$ & temporal \\
SPK & PQK $_{\mathrm{SPK}}$ & $\epsilon_{1}=1, \epsilon_{2}= \pm 1$ & Euclidean \\
\hline
\end{tabular}

Table 4. As far as the scalar geometries are concerned, there are three distinct c-maps. The parameters refer to the hypermultiplet 'master Lagrangian' (2.2).

a SPK base (the space parametrized by the para-complex scalars $z^{\alpha}$ ). Finally, the manifolds with $\left(\epsilon_{1}=1, \epsilon_{2}= \pm 1\right)$ are isometric (keeping the base manifold fixed). Thus there are three inequivalent cases: QK, PQK with an SK base and PQK with an SPK base, see table 4 for a summary. If we need to empasize the base we will write $\mathrm{PQK}_{\mathrm{SK}}$ or $\mathrm{PQK}_{\mathrm{SPK}}$. We remark that the c-maps $\mathcal{M}_{2 n_{V}} \rightarrow \mathcal{N}_{4 n_{V}+4}$ are maps between $\mathrm{S}(\mathrm{P}) \mathrm{K}$ manifolds and $\mathrm{Q}(\mathrm{P}) \mathrm{K}$ manifolds, which are well defined on their own, that is without reference to supermultiplets, Lagrangians and dimensional reduction. In particular the resulting QK/PQK manifolds are admissible (though non-generic) HM target manifolds in all signatures for dimensions up to six, provided that they are compatible with the R-symmetry group. We will see that QK/PQK manifolds of all of these types appear in type-II compactifications on Calabi-Yau threefolds.

\subsection{Reduction to three dimensions}

Let us consider the dimensional reduction of $\mathcal{N}=2$ supergravity with $n_{V}$ vector multiplets and $n_{H}$ hypermultiplets to three dimensions. The field content and scalar geometry of the HM sector does not change, while vector multiplets can be dualized into hypermultiplets after reduction. Moreover, the bosonic degrees of freedom of the supergravity multiplet, that is the metric and the graviphoton, give rise to an additional hypermultiplet, so that we end up with three-dimensional $\mathcal{N}=4$ supergravity with $\left(n_{V}+1\right)+n_{H}$ hypermultiplets. Since the four-dimensional HMs play a passive role, we only need to consider the bosonic Lagrangian (2.1) for gravity and $n_{V}$ vector multiplets. There are four different starting points: signature $(1,3)$ with either the standard or twisted $\mathcal{N}=2$ algebra, signature $(0,4)$ and signature $(2,2)$. The scalar geometry and relative signs are encoded in two parameters: $\epsilon_{1}=\mp 1$ distinguishes between SK (signature $(1,3)$ ) and SPK (signatures $(0,4),(2,2)$ ), while $\lambda= \pm 1$ encodes the relative sign between scalar and vector terms. As mentioned earlier, the choice of this sign is only relevant in signature $(1,3)$, since in the other signatures it can be changed by a field redefinition. We introduce another parameter $\epsilon=\mp 1$, which distinguishes between spacelike reduction and timelike reduction. After the reduction, the Einstein-Hilbert term is non-dynamical, and the local degrees of freedom of the four-dimensional metric reside in the KK-scalar $\varphi$ and the scalar $\tilde{\phi}$ which is dual to the KK-vector. The four-dimensional vector fields $A_{\mu}^{I}$ decompose into scalars $\zeta^{I}$ and three-dimensional vector fields which we dualize into scalars $\tilde{\zeta}_{I}$. Together with the (para-) complex scalars $z^{\alpha}$, this is the field content of $n_{H}+1$ hypermultiplets. The computation is the same as in [29] and [26], except that we now include the case $\lambda=+1$. The Lagrangian 
takes the form

$$
\begin{aligned}
\mathbf{e}^{-1} L_{3}= & \frac{1}{2} R_{3}-\frac{1}{4} \partial_{\mu} \phi \partial^{\mu} \phi-g_{\alpha \bar{\beta}} \partial_{\mu} z^{\alpha} \partial^{\mu} \bar{z}^{\bar{\beta}} \\
& +\epsilon_{1} e^{-2 \phi}\left[\partial^{\rho} \tilde{\phi}+\frac{1}{2}\left(\zeta^{I} \partial^{\rho} \tilde{\zeta}_{I}+\tilde{\zeta}_{I} \partial^{\rho} \zeta^{I}\right)\right]\left[\partial_{\rho} \tilde{\phi}+\frac{1}{2}\left(\zeta^{I} \partial_{\rho} \tilde{\zeta}_{I}-\tilde{\zeta}_{\mathcal{I}} \partial_{\rho} \zeta^{I}\right)\right] \\
& +\frac{\lambda \epsilon}{2} e^{-\phi}\left[\mathcal{I}_{I J} \partial_{\mu} \zeta^{I} \partial^{\mu} \zeta^{J}-\epsilon_{1} \mathcal{I}^{I J}\left(\partial^{\rho} \tilde{\zeta}_{I}-\mathcal{R}_{I K} \partial^{\rho} \zeta^{K}\right)\left(\partial_{\rho} \tilde{\zeta}_{J}-\mathcal{R}_{J L} \partial_{\rho} \zeta^{L}\right)\right] .
\end{aligned}
$$

By comparison to $(2.2)$ we read off that $\lambda \epsilon=-\epsilon_{2}$. All of these spaces are either QK, PQK $\mathrm{SK}_{\mathrm{S}}$ or $\mathrm{PQK}_{P \mathrm{SK}}$. Starting with four theories in four dimensions, we have six different cases.

1. Start with VMs in signature $(1,3)$, with the standard $\mathcal{N}=2$ algebra, and reduce over space, $(1,3) \rightarrow(1,2)$. Then $\epsilon_{1}=-1$ and $\epsilon=-1, \lambda=-1$ which implies $\epsilon_{2}=-1$. This is the standard ('spatial') c-map of [29] which maps $\mathrm{SK}_{+} \rightarrow$ QK.

2. Start with VMs in signature $(1,3)$, with the standard $\mathcal{N}=2$ algebra, and reduce over time, $(1,3) \rightarrow(0,3)$. Then $\epsilon_{1}=-1$ and $\epsilon=1, \lambda=-1$ which implies $\epsilon_{2}=1$. This is the temporal c-map [26], which maps $\mathrm{SK}_{+} \rightarrow \mathrm{PQK}_{\mathrm{SK}}$.

3. Start with VMs in signature $(0,4)$ and reduce over space, $(0,4) \rightarrow(0,3)$. Then $\epsilon_{1}=1$ and $\epsilon=-1, \lambda= \pm 1$ which implies $\epsilon_{2}= \pm 1$. This is the Euclidean c-map [26], which maps $\mathrm{SPK} \rightarrow \mathrm{PQK}_{\mathrm{SPK}}$.

4. Start with VMs in signature $(2,2)$ and reduce over time, $(2,2) \rightarrow(1,2)$. Then $\epsilon_{1}=1$ and $\epsilon=1, \lambda= \pm 1$ which implies $\epsilon_{2}= \pm 1$. This works like the Euclidean c-map, $\mathrm{SPK} \rightarrow \mathrm{PQK}_{\mathrm{SPK}}$, but if we want to emphasize the context of dimensional reduction, that is, that we reduce over time rather than space, we will call it the neutral c-map.

5. Start with VMs in signature $(1,3)$, with the twisted $\mathcal{N}=2$ algebra, and reduce over space, $(1,3) \rightarrow(1,2)$. Then $\epsilon_{1}=-1$ and $\epsilon=-1, \lambda=1$ which implies $\epsilon_{2}=-1$. This maps SK to PQK with a SK base: $\mathrm{SK}_{-} \rightarrow \mathrm{PQK}_{\mathrm{SK}}$. Thus the sign flip between scalar and vector fields exchanges the roles of the spatial and temporal c-map. In the case at hand we obtain a PQK manifold from an SK manifold through spatial reduction. While this works like the temporal c-map as far as the scalar geometries are concerned, we will call this the twisted spatial c-map if we want to emphasize the context of dimensional reduction, that is, that we reduce over space, but start with flipped four-dimensional gauge kinetic terms.

6. Start with VMs in signature $(1,3)$, with the twisted $\mathcal{N}=2$ algebra, and reduce over time, $(1,3) \rightarrow(0,3)$. Then $\epsilon_{1}=-1$ and $\epsilon=1, \lambda=1$ which implies $\epsilon_{2}=1$. This maps SK to QK despite that we are reducing over time: $\mathrm{SK}_{-} \rightarrow$ QK. While this works like the spatial c-map as far as the manifolds are concerned, we will call this the twisted temporal c-map if we need to emphasize the context of dimensional reduction.

See table 5 for a summary. 


\begin{tabular}{|c|c|c|c|c|}
\hline $4 \mathrm{~d}$ signature & Source & $3 \mathrm{~d}$ signature & Image & c-map \\
\hline$(0,4)$ & SPK & $(0,3)$ & $\mathrm{PQK}_{\mathrm{SPK}}$ & Euclidean \\
\hline$(1,3)$ & $\mathrm{SK}_{+}$ & $(0,3)$ & $\mathrm{PQK}_{\mathrm{SK}}$ & temporal \\
\hline$(1,3)$ & $\mathrm{SK}_{-}$ & $(0,3)$ & QK & twisted temporal $\cong$ spatial \\
\hline$(1,3)$ & $\mathrm{SK}_{+}$ & $(1,2)$ & QK & spatial \\
\hline$(1,3)$ & $\mathrm{SK}_{-}$ & $(1,2)$ & $\mathrm{PQK}_{\mathrm{SK}}$ & twisted spatial $\cong$ temporal \\
\hline$(2,2)$ & SPK & $(1,2)$ & $\mathrm{PQK}_{\mathrm{SPK}}$ & neutral $\cong$ Euclidean \\
\hline
\end{tabular}

Table 5. When reducing four-dimensional vector multiplets to three dimensions, there are six distinct cases, although there are only three distinct types of hypermultiplet manifolds that arise from the construction.

If we start with a theory of $n_{V}$ vector and $n_{H}$ hypermultiplets in four dimensions, with scalar manifold $\mathcal{M}_{2 n_{V}} \times \tilde{\mathcal{N}}_{4 n_{H}}$, reduction to three dimensions leads us to a theory with $\left(n_{V}+1\right)+n_{H}$ hypermultiplets, where the two hypermultiplet manifolds form a direct product:

$$
\mathcal{M}_{2 n_{V}} \times \tilde{\mathcal{N}}_{4 n_{H}} \rightarrow \mathcal{N}_{4 n_{V}+4} \times \tilde{\mathcal{N}}_{4 n_{H}}
$$

If both factors are 'in the image of the c-map', the three-dimensional theory can be lifted to a different four-dimensional theory with $n_{V}^{\prime}=n_{H}-1$ vector multiplets and $n_{H}^{\prime}=n_{V}+1$ hypermultiplets.

$$
\mathcal{M}_{2 n_{V}} \times \tilde{\mathcal{N}}_{4 n_{H}} \rightarrow \tilde{\mathcal{N}}_{4 n_{V}+4} \times \tilde{\mathcal{N}}_{4 n_{H}} \leftarrow \tilde{\mathcal{N}}_{4 n_{V}+4} \times \tilde{\mathcal{M}}_{2 n_{H}-2}=\tilde{\mathcal{N}}_{4 n_{H}^{\prime}} \times \tilde{\mathcal{M}}_{2 n_{V}^{\prime}}
$$

In the context of string theory, the relations between the four-dimensional theories are T-dualities, which we call spacelike, timelike and mixed depending on how they combine spacelike/timelike reduction with spacelike/timelike oxidation. Which T-dualities exist depends on the details of the HM sectors of the four-dimensional theories. Therefore we will now consider the Calabi-Yau compactifications of type-II theories in signature $(0,10),(1,9)$ and $(2,8)$, which give rise to four-dimensional $\mathcal{N}=2$ theories in signatures $(0,4),(1,3)$ and $(2,2)$.

\section{Ten-dimensonal type-II string theories}

As is well known, type-IIA and type-IIB string theory are related by T-duality. When admitting timelike T-duality, one obtains two further theories, dubbed type-IIA*/IIB*, as summarized in figure 1 [1].

The difference between type-IIA and type-IIA* and between type-IIB and type-IIB* lies in certain phase factors, which at the level of the effective supergravity Lagrangian manifest themselves in sign flips of the kinetic terms of the R-R fields, as well as factors of powers of $i$ in the fermionic terms. For type-IIB/IIB* the scalar manifolds are different, namely $\mathrm{SL}(2, \mathbb{R}) / \mathrm{SO}(2)$ for type-IIB and $\mathrm{SL}(2, \mathbb{R}) / \mathrm{SO}(1,1)$ for type-IIB*. It was observed that supersymmetry is realized in type-II* theories in a modified, twisted form, which can be interpreted as a generalized $O(p, q)$ Majorana condition [2]. In [10] the R-symmetry groups for supersymmetry algebras in arbitrary dimension and signature were classified, 


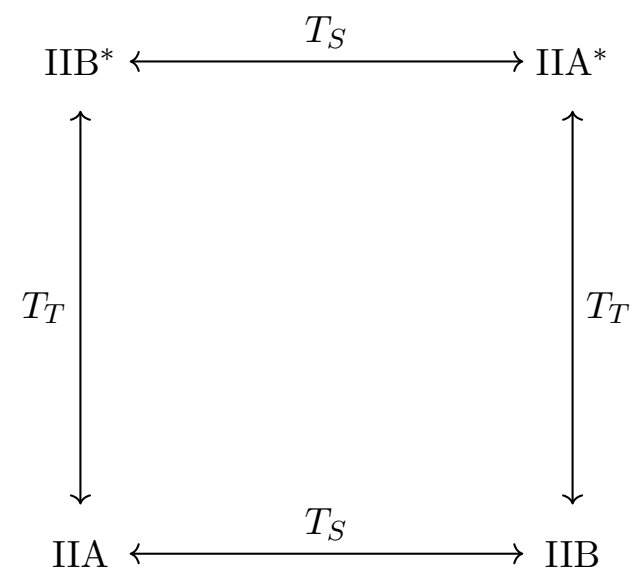

Figure 1. Diagram showing the relationship between II and $\mathrm{II}^{*}$ theories, where $T_{S}\left(T_{T}\right)$ denotes a spacelike (timelike) T-duality.

which allows to put this observation into a wider context. It was found that in certain signatures there exist several non-isomorphic supersymmetry algebras with the same number of supercharges (and, where applicable the same chirality properties), whose R-symmetry groups are different real forms of the same complex Lie group. For example, in signature $(1,9)$, chiral supersymmetry algebras with $\mathcal{N}$ left-moving (or right-moving) supercharges are real forms of a complex supersymmetry algebra with R-symmetry group $O(\mathcal{N}, \mathbb{C})$. Real supersymmetry algebras are obtained by imposing $O(p, q)$ Majorana conditions, with $p+q=\mathcal{N}$, which leads to real supersymmetry algebras with R-symmetry group $O(p, q)$. For ten-dimensional chiral supersymmetry algebras with 32 real supercharges the two possible cases are $O(2)$ and $O(1,1)$ which correspond to type-IIB/IIB*. There also are two inequivalent non-chiral algebras, which have the same discrete R-symmetry group but differ by a relative sign in the reality condition imposed on left- and right-moving supercharges, corresponding to type-IIA/IIA*. Similarly, $\mathcal{N}$-extended supersymmetry algebras in fourdimensions are real forms of a complex supersymmetry algebra with R-symmetry $\operatorname{GL}(\mathcal{N}, \mathbb{C})$ and the reality conditions defining real supersymmetry algebras in signature $(1,3)$ lead to R-symmetry groups of the form $\mathrm{U}(p, q), p+q=\mathcal{N}$. For $\mathcal{N}=2$ the two possibilities are $\mathrm{U}(2)$ and $\mathrm{U}(1,1)$. For completeness we note that for $\mathcal{N}=2$ the reality conditions defining real supersymmetry algebras in signatures $(0,4)$ and $(2,2)$ lead to unique algebras with R-symmetry $\mathrm{U}^{*}(2)$ and $\mathrm{GL}(2, \mathbb{R})$, respectively, see [10] for details.

All type-II theories have the same NS-NS sector which consists of the graviton $G_{M N}$, Kalb-Ramond field $B_{M N}$ and dilaton $\Phi$. The R-R sector of type-IIA/IIA* contains a one form $C_{1}$ and a three-form $C_{3}$ while the R-R sector of type-IIB/IIB* contains a zero-form $C_{0}$, a two-form $C_{2}$ and a four-form $C_{4}$ whose field strength is self-dual or anti-self-dual, $* G_{5}= \pm G_{5} .{ }^{5}$ The difference between the bosonic actions of type-II and type-II* is a sign flip of the kinetic terms for all fields in R-R sector, see tables 6 and $7 .^{6}$

\footnotetext{
${ }^{5}$ We will specify our choice of sign below.

${ }^{6}$ The information for type-IIA is taken from table 1 in [2]. Note that their notation for signature is $(s, t)$, where $s$ corresponds to positive eigenvalues of the metric, and $t$ to negative eigenvalues of the metric, while we use $(t, s)$.
} 


\begin{tabular}{|l|l|l|l|l|l|}
\hline Type & $G_{M N}$ & $B_{M N}$ & $\Phi$ & $C_{1}$ & $C_{3}$ \\
\hline $\operatorname{IIA}_{(1,9)}$ & + & + & + & + & + \\
$\operatorname{IIA}_{(1,9)}^{*}$ & + & + & + & - & - \\
$\operatorname{IIA}_{(0,10)}$ & + & - & + & - & + \\
$\operatorname{IIA}_{(2,8)}$ & + & - & + & + & - \\
\hline
\end{tabular}

Table 6. Relative signs for kinetic terms in ten-dimensional type-IIA theories. A + sign corresponds to a standard kinetic term in Lorentz signature, thus discarding the overall - sign with which these terms appear in the action when using the mostly plus convention for the metric.

\begin{tabular}{|l|l|l|l|l|l|l|}
\hline Type & $G_{M N}$ & $B_{M N}$ & $\Phi$ & $C_{0}$ & $C_{2}$ & $C_{4}$ \\
\hline $\operatorname{IIB}_{(1,9)}$ & + & + & + & + & + & + \\
$\operatorname{IIB}_{(1,9)}^{*}$ & + & + & + & - & - & - \\
$\operatorname{IIB}_{(1,9)}^{\prime}$ & + & - & + & - & + & - \\
\hline
\end{tabular}

Table 7. Relative signs for kinetic terms in ten-dimensional type-IIB theories. A + indicates the standard sign for a theory in Lorentz signature using the mostly plus convention.

The bosonic actions for type-IIA/IIA* take the form [2]

$$
S_{(1,9)}^{\mathrm{IIA} / \mathrm{IIA}^{*}}=\int d^{10} x \sqrt{|G|} e^{-2 \Phi}\left(R+4(\partial \Phi)^{2}-H^{2}+\lambda G_{2}^{2}+\lambda G_{4}^{2}\right)+\cdots
$$

where we omitted the Chern-Simons terms, and where $H, G_{2}, G_{4}$ are the field strength of $B, C_{1}, C_{3}$, respectively. Type-IIA corresponds to $\lambda=-1$ while type-IIA* corresponds to $\lambda=1$. Taking into account that we use the mostly plus convention for the metric, this means that all bosonic fields have positive kinetic energy for $\lambda=-1$. In table 6 this corresponds to a row where all entries are + , that is we discard the overall minus sign that these terms have in the action. Generally, in this and other tables, we record sign flips relative to standard kinetic terms in Lorentz signature, which correspond to a row with only + signs. Note that the kinetic term of the dilaton in the action (3.1) has a + sign, since we are in the string frame. When going to the Einstein frame, this sign flips, showing that the dilaton has positive kinetic energy.

For type-IIB/IIB* there is no simple covariant action, since the five-form field strength is self-dual. However one can use a pseudo-action, whose variation gives the field equation except the self-duality condition $G_{5}= \pm * G_{5}$, which is then imposed by hand [2]:7

$$
S_{(1,9)}^{\mathrm{IIB} / \mathrm{IIB}^{*}}=\int d^{10} x \sqrt{|G|} e^{-2 \Phi}\left(R+4(\partial \Phi)^{2}-H^{2}+\lambda G_{1}^{2}+\lambda G_{3}^{2}+\lambda G_{5}^{2}\right)+\cdots
$$

\footnotetext{
${ }^{7}$ The sign in the self-duality relation is correlated with the sign of terms which we have not displayed (Chern-Simons and fermionic terms), see for example [43] for a general discussion. Full bosonic type-II Lagrangians, which however use a different notation and normalization for the bosonic fields, can be found in [4]. In their conventions the sign is correlated with whether the worldvolume theories of fundamental strings and D-strings are Lorentzian or Euclidean, resulting in a (+)-sign for type-IIB and a (-)-sign for type-IIB*/IIB'.
} 
where $G_{p+1}=d C_{p}+\cdots$ are the field strength and where again we only display the Maxwelllike terms. Type-IIB corresponds to $\lambda=-1$, where all kinetic terms have their standard sign, while type-IIB* exhibits a sign flip for all R-R fields.

Type-II string theories exist for all ten-dimensional signatures. We will use the notation type- $\mathrm{II}_{(t, s)}$ for a theory where the metric has $t$ negative and $s$ positive eigenvalues. Since we prefer the mostly plus convention for Lorentz signature, we will usually refer to the $t$ directions as timelike and the $s$ directions as spacelike. Theories where $t$ and $s$ are exchanged have been shown to be equivalent [2]. The unique theories in signatures $(0,10)$ and $(2,8)$ are IIA theories, denoted $\operatorname{IIA}_{(0,10)}$ and $\operatorname{IIA}_{(2,8)}$. These theories are non-chiral and have the same R-R sector as type-IIA $(1,9)$. Their actions have the same structure as the type-IIA $(1,9)$ action, but with some sign flips for the Maxwell-like terms [2], which are listed in table 6 . In both signatures the $B$-field has a flipped kinetic term, while in the R-R sector either $C_{1}$ or $C_{3}$ has a sign flip:

$$
\begin{aligned}
S_{(0,10)}^{\mathrm{IIA}} & =\int d^{10} x \sqrt{|G|} e^{-2 \Phi}\left(R+4(\partial \Phi)^{2}+H^{2}+G_{2}^{2}-G_{4}^{2}\right)+\cdots, \\
S_{(2,8)}^{\mathrm{IIA}} & =\int d^{10} x \sqrt{|G|} e^{-2 \Phi}\left(R+4(\partial \Phi)^{2}+H^{2}-G_{2}^{2}+G_{4}^{2}\right)+\cdots .
\end{aligned}
$$

Type-II string theories in different signatures are related by what we call mixed T-dualities, that is T-dualities which combine a spacelike/timelike reduction with a timelike/spacelike oxidation (lifting). For this to work one needs to make use of the S-dual of the type-IIB* theory, which is called type-IIB'. As shown in [2] Buscher T-duality along an isometric direction $X^{\sharp}$ in the target space of the worldsheet sigma model preserves the sign of the term $G_{\sharp \sharp} \partial_{\alpha} X^{\sharp} \partial^{\alpha} X^{\sharp}$ if the worldsheet theory has Lorentzian signature, but reverses it if the worldsheet theory has Euclidean signature. In the type-IIB*-theory, D-branes are replaced by E-branes, which have a Euclidean worldvolume. If one applies S-duality, fundamental IIB*-strings and E-strings are exchanged, so that in the resulting IIB'-theory fundamental strings have a Euclidean worldvolume. The type-IIA $(0,10)$ and type-IIA $(2,8)$ theories are then obtained from the type-IIB' ${ }_{(1,9)}$ theory by T-dualities which involve a timelike/spacelike reduction combined by a spacelike/timelike oxidation [2]. At the supergravity level, S-duality exchanges the $B$-field and the R-R two-form $C_{2}$, resulting in the sign flips recorded in table 7 ,

$$
S_{(1,9)}^{\mathrm{IIB}^{\prime}}=\int d^{10} x \sqrt{|G|} e^{-2 \Phi}\left(R+4(\partial \Phi)^{2}+H^{2}+G_{1}^{2}-G_{3}^{2}+G_{5}^{2}\right)+\cdots
$$

Note that while $G_{5}$ is an S-duality singlet, $\Phi$ and $C_{0}$ parametrize the indefinite signature coset space $\mathrm{SL}(2, \mathbb{R}) / \mathrm{SO}(1,1)$ on which S-duality acts non-linearly.

\section{Type-II Calabi-Yau compactifications}

By compactification of type-II string theories one obtains $\mathcal{N}=2$ supergravity coupled to vector and hypermultiplets. Since we always compactify six spatial dimensions to go from signatures $(0,10),(1,9),(2,8)$ to signatures $(0,4),(1,3),(2,2)$, the only essential difference in these reductions is between type-IIA and type-IIB, which are distinguished, as far as bosonic 
degrees of freedom are concerned, by the field content of their R-R sectors. Otherwise the bosonic type-IIA/IIA* actions only differ from one another by relative sign flips that one has to follow through, and the same applies to the type-IIB/IIB*/IIB' theories. Since the mechanics of Calabi-Yau compactifications is well known from the standard cases of $\operatorname{IIA}_{(1,9)}[16]$ and $\operatorname{IIB}_{(1,9)}$ [17], we will not go through the computational details but highlight how the ten-dimensional sign flips modify the resulting four-dimensional actions. More details are given in the appendix. The reduction of the Euclidean $\operatorname{IIA}_{(0,10)}$ theory was worked out in detail in [18].

\subsection{Type-IIA Calabi-Yau compactifications}

We start with type-IIA theories, where we have the cases type-IIA $(1,9), \operatorname{IIA}_{(1,9)}^{*}, \operatorname{IIA}_{(0,10)}$ and $\operatorname{IIA}_{(2,8)}$. We first consider aspects which work the same in all cases.

The metric. In a general real six-fold compactification, the massless four-dimensional fields resulting from the reduction of the metric $G_{M N}$ are the four-dimensional metric $g_{\mu \nu}$, vector fields, and scalar fields. Massless vector fields are on one-to-one correspondence with Killing vector fields, and since CY3-folds (Calabi-Yau threefolds) do not have isometries, there are no massless vectors in our case. The massless scalar fields are in one-to-one with deformations of the six-fold metric which preserve Ricci-flatness. For CY3-folds these deformations are, due to the existence of a holomorphic $(3,0)$-form, in one-to-one correspondence with the deformations of the complex structure and of the (real) Kähler form. This gives rise to $h^{2,1}$ complex scalars $z^{\alpha}, \alpha=1, \ldots, h^{2,1}$ and $h^{1,1}$ real scalars $y^{A}$, $A=1, \ldots, h^{1,1}$, where $h^{i, j}$ are the Hodge numbers of the CY3-fold.

$\boldsymbol{p}$-form fields. A ten-dimensional $p$-form decomposes into products of four-dimensional $p^{\prime}$-forms and six-dimensional $p^{\prime \prime}$-forms, where $p^{\prime}+p^{\prime \prime}=p$. Massless $p^{\prime}$-forms are in one-to-one correspondence with harmonic $p^{\prime \prime}$-forms, which are counted by the Betti-numbers $b_{p^{\prime \prime}}$ of the compact space. For a CY3-fold the Betti numbers are related to the Hodge numbers by $b_{p^{\prime \prime}}=\sum_{i+j=p^{\prime \prime}} h^{i, j}$. Moreover, for a CY3-fold

$$
h^{0,0}=h^{3,0}=h^{0,3}=h^{3,3}=1, \quad h^{1,0}=h^{0,1}=h^{3,2}=h^{2,3}=0, \quad h^{3,0}=h^{0,3}=1,
$$

so that the only numbers that vary between CY3s are $h^{1,1}=h^{2,2} \geq 1$ and $h^{1,2}=h^{2,1} \geq 0$.

The B-field. The $B$-field $B_{M N}$ gives rise to $h^{1,1}$ real scalars $x^{A}$, as well as a fourdimensional $B$-field, which we dualize into a scalar $\tilde{\phi}$.

The dilaton. The ten-dimensional dilaton $\Phi$ gives rise to a four-dimensional scalar $\varphi$, which differs from $\Phi$ by a field-redefinition. Essentially, one absorbs a factor proportional to the volume of the internal space, in order that the four-dimensional action acquires standard form.

The R-R sector. The R-R one-form $C_{M}$ gives rise to a vector $\mathcal{A}_{\mu}^{0}$. The R-R three-form $C_{M N P}$ gives rise to $h^{1,1}$ vectors $\mathcal{A}_{\mu}^{A}$ and $2 h^{2,1}+2$ real scalars $\zeta^{I}, \tilde{\zeta}_{I}, I=0,1, \ldots, h^{2,1}$. The massless fields originating from the NS-NS sector are summarized in table 8 , those from the R-R sector in table 9. 


\begin{tabular}{|l|l|l|}
\hline $10 \mathrm{~d}$ & $4 \mathrm{~d}$ & \\
\hline$G_{M N}$ & $g_{\mu \nu}$ & Metric \\
& $z^{\alpha}$ & Complex structure moduli \\
& $y^{A}$ & (Real) Kähler moduli \\
\hline$B_{M N}$ & $b_{\mu \nu} \sim \tilde{\phi}$ & Universal axion \\
& $x^{A}$ & $h^{1,1}$ real scalars \\
\hline$\Phi$ & $\varphi$ & Dilaton \\
\hline
\end{tabular}

Table 8. Massless fields in type-II Calabi-Yau compactifications, NS-NS sector.

\begin{tabular}{|l|l|l|}
\hline $10 \mathrm{~d}$ & $4 \mathrm{~d}$ & \\
\hline$C_{M}$ & $\mathcal{A}_{\mu}^{0}$ & vector \\
\hline$C_{M N P}$ & $C_{\mu n p} \sim \mathcal{A}_{\mu}^{A}$ & $h^{1,1}$ vectors \\
& $C_{m n p} \sim \zeta^{I}, \tilde{\zeta}_{I}$ & $2 h^{2,1}+2$ scalars \\
\hline
\end{tabular}

Table 9. Massless fields in type-IIA Calabi-Yau compactifications, R-R sector.

\begin{tabular}{|l||l|l||l|l|l|l|l||l|l|}
\hline & $y^{A}$ & $x^{A}$ & $z^{\alpha}$ & $\varphi$ & $\tilde{\phi}$ & $\zeta^{I}$ & $\tilde{\zeta}_{I}$ & $\mathcal{A}_{\mu}^{0}$ & $\mathcal{A}_{\mu}^{A}$ \\
\hline $\operatorname{IIA}_{(1,9)}$ & + & + & + & + & + & + & + & + & + \\
$\operatorname{IIA}_{(1,9)}^{*}$ & + & + & + & + & + & - & - & - & - \\
$\operatorname{IIA}_{(0,10)}$ & + & - & + & + & + & + & + & - & + \\
$\operatorname{IIA}_{(2,8)}$ & + & - & + & + & + & - & - & + & - \\
\hline
\end{tabular}

Table 10. Signs of the kinetic terms for scalar and vector fields resulting from type-IIA CY3 compactifications. A + indicates a standard kinetic term. The fields $y^{A}, x^{A}$ are the vector multiplet scalars.

Collecting all these fields, this is the bosonic field content of the $\mathcal{N}=2$ Poincaré supergravity multiplet, $\left(g_{\mu \nu}, \mathcal{A}_{\mu}^{0}\right)$, of $n_{V}=h^{1,1}$ vector multiplets $\left(y^{A}, x^{A}, \mathcal{A}_{\mu}^{A}\right)$, and of $n_{H}=h^{2,1}+1$ hypermultiplets $\left(z^{\alpha}, \varphi, \tilde{\phi}, \zeta^{I}, \tilde{\zeta}_{I}\right)$. The signs of the kinetic terms of the scalar fields can be inferred from those of the higher-dimensional ones, and are listed in table 10.

Signs are taken relative to the standard IIA $_{(1,9)}$ theory, where all kinetic terms have the standard sign, denoted + . In $\operatorname{IIA}_{(1,9)}^{*}$ half of the signs in the HM sector are flipped, so that the HM scalar manifold has neutral signature. In the Euclidean IIA $(0,10)$ theory only the signs of the scalars $x^{A}$ which descend from the $B$-field are flipped, which gives the VM manifold neutral signature. Finally in the $\operatorname{IIA}_{(2,8)}$ case, we have signs flips for $x^{A}, \zeta^{I}, \tilde{\zeta}_{I}$, so that both VM and HM scalar manifold have neutral signature. ${ }^{8}$

The ten-dimensional sign flips also affect the four-dimensional vector kinetic terms. For type-IIA $_{(1,9)}^{*}$ the signs of all vector kinetic terms are flipped, whereas for type-IIA $(0,10)$ only

\footnotetext{
${ }^{8}$ Note that in both cases the sign flip of the four-dimensional $B$-field $b_{\mu \nu}$ is compensated by a second sign flip when we dualize this two-form into the scalar $\tilde{\phi}$. Dualization flips the sign of the kinetic term for a $p$-form field if and only if the metric has an even number of negative eigenvalues.
} 
the sign of $\mathcal{A}_{\mu}^{0}$ is flipped, while for type-IIA $(2,8)$ only the signs of $\mathcal{A}_{\mu}^{A}$ are flipped. Thus the vector kinetic terms have signatures $(+)^{h^{1,1}+1},(-)^{h^{1,1}+1},(+)^{h_{1,1}}(-)$, and $(+)(-)^{h^{1,1}}$, respectively.

The interactions of these fields are encoded in certain coupling matrices that one obtains when performing the dimensional reduction. For a four-dimensional $\mathcal{N}=2$ theory these coupling matrices can be interpreted as geometrical data on the scalar manifolds $\mathcal{M}_{2 h^{1,1}}$ of the vector and $\mathcal{N}_{4 h^{2,1}+4}$ of hypermultiplets, which have real dimensions $2 h^{1,1}$ and $4 h^{2,1}+4$, respectively. These are the geometries that we have reviewed in the previous section. At this point the sign flips become relevant since they determine the signatures of the metrics of $\mathcal{M}_{2 h^{1,1}}$ and $\mathcal{N}_{4 h^{2,1}+4}$.

\subsubsection{The vector multiplet sector}

Let us first consider the vector multiplet scalars $y^{A}$ and $x^{A}$. In signature $(1,9)$ their kinetic terms come with same sign, and the manifold $\mathcal{M}_{2 h^{1,1}}$ can be shown to be a complex manifold. The real scalars $y^{A}$ and $x^{A}$ can be combined into complex scalars $z^{A}=y^{A}+i x^{A}$, which provide holomorphic coordinates for $\mathcal{M}_{2 h^{1,1}}$. The scalar fields $y^{A}$ parametrize the moduli space of real Kähler forms $J$ on the CY3, while $x^{A}$ parametrize the deformations of the internal components of the $B$-field, which corresponds to a harmonic (1,1)-form on the CY3. The combined moduli space parametrized by $z^{A}$ can be viewed as a complexification of the real moduli space of Kähler forms, and is usually just called the Kähler moduli space. This space carries itself a Kähler metric $g_{A \bar{B}}(z, \bar{z})$, which appears in the four-dimensional action as the generalized kinetic term (sigma model) of the scalars $z^{A}$, that is $\mathcal{L} \sim g_{A \bar{B}}(z, \bar{z}) \partial_{\mu} z^{A} \partial^{\mu} \bar{z}^{\bar{B}}$. Moreover, this Kähler metric is not generic, but special, because its Kähler potential $K(z, \bar{z})$ can be obtained from a holomorphic prepotential $\mathcal{F}(z)$. Thus $g_{A \bar{B}}(z, \bar{z})$ is a (projective) special Kähler metric or SK metric for short.

Let us next look at the four-dimensional vector fields, still restricting ourselves to signature $(1,9)$. We have obtained $h^{1,1}+1$ vector fields, of which one, $\mathcal{A}_{\mu}^{0}$, belongs to the supergravity multiplet and is called the graviphoton, while the others, $\mathcal{A}_{\mu}^{A}$, belong to the $h^{1,1}$ vector multiplets. We denote the corresponding field strength by $\mathcal{F}_{\mu \nu}^{0}$ and $\mathcal{F}_{\mu \nu}^{A}$. As explained before, the vector fields can be rearranged into linear combinations $A_{\mu}^{\Sigma}, \Sigma=0, \ldots, n_{V}=h^{1,1}$ so that the field strength $F_{\mu \nu}^{\Sigma}$ together with their duals $G_{\Sigma \mid \mu \nu}$ form a symplectic vector. By carrying out the reduction explicitly, one finds that the couplings between scalars and vectors are encoded by the complex coupling matrix $\mathcal{N}_{\Sigma \Lambda}=\mathcal{R}_{\Sigma \Lambda}+i \mathcal{I}_{\Sigma \Lambda}$, which depends on the scalars $z^{A}$ through the prepotential $\mathcal{F}\left(z^{A}\right)$.

The resulting bosonic Lagrangian for the supergravity multiplet and $n_{V}$ vector multiplets has the form (2.1), and the only difference between type-IIA and type-IIA* is the overall sign flip for the vector fields $A_{\mu}^{\Sigma}$. Since we use a convention where $\mathcal{I}_{\Sigma \Lambda}$ is negative definite, type-IIA corresponds to $\lambda=-1$, while type-IIA* corresponds to $\lambda=1$ :

$$
L_{G+\mathrm{VM}}^{(1,3) \mathrm{IIA} / \mathrm{IIA}^{*}}=\frac{1}{2} \star R_{4}-\bar{g}_{A \bar{B}}(z, \bar{z}) d z^{A} \wedge \star d \bar{z}^{B}-\frac{\lambda}{4} \mathcal{I}_{\Sigma \Lambda} F^{\Sigma} \wedge \star F^{\Lambda}+\frac{1}{4} \mathcal{R}_{\Sigma \Lambda} F^{\Sigma} \wedge F^{\Lambda}
$$


where $A, B=1, \ldots, n_{V}=h^{1,1}$ and $\Lambda, \Sigma=0, \ldots, n_{V}=h^{1,1}$. For $\lambda=-1$ this is the standard result of [16]. For the type-IIA* the sign flips in the ten-dimensional Lagrangian induce a sign flip in the four-dimensional Maxwell term.

In signatures $(0,10)$ and $(2,8)$ table 10 shows that the metric of $\mathcal{M}_{2 h^{1,1}}$ has neutral signature. The four-dimensional $\mathcal{N}=2$ supersymmetry algebra requires SPK geometry for the vector multiplets in these cases. The case $(0,10) \rightarrow(0,4)$ has been worked out in full detail in [18]. As far as the vector multiplet sector is concerned, the only difference between this and the case $(2,8) \rightarrow(2,2)$ is an overall sign flip of the Maxwell term. Note that in both cases the vector kinetic terms have Lorentz signature and therefore are indefinite. As mentioned before, the overall sign of the Maxwell term is conventional in the sense that it can be flipped by a field redefinition. Therefore we can take either value of $\lambda= \pm 1$ in the following Lagrangian:

$$
L_{G+\mathrm{VM}}^{(0,4),(2,2)}=\frac{1}{2} \star R_{4}-\bar{g}_{A \bar{B}}(z, \bar{z}) d z^{A} \wedge \star d \bar{z}^{B}+\frac{\lambda}{4} \mathcal{I}_{\Sigma \Lambda} F^{\Sigma} \wedge \star F^{\Lambda}+\frac{1}{4} \mathcal{R}_{\Sigma \Lambda} F^{\Sigma} \wedge F^{\Lambda} .
$$

Compared to (4.1) the scalar geometry is now SPK and the scalar fields $z^{A}$ are paracomplex fields. The couplings $\bar{g}_{A \bar{B}}, \mathcal{I}_{\Sigma \Lambda}$ and $\mathcal{R}_{\Sigma \Lambda}$ are determined by the standard formulae of special geometry, but using a para-holomorphic instead of a holomorphic prepotential, see $[18,24,25]$ for details.

\subsubsection{The hypermultiplet sector}

The scalars $z^{\alpha}, \alpha=1, \ldots, h^{2,1}$ parametrize the deformations of the complex structure of the CY3 metric. They provide coordinates on a special Kähler submanifold of the hypermultiplet manifold, with metric $g_{\alpha \bar{\beta}}(z, \bar{z})$ and prepotential $\mathcal{F}\left(z^{\alpha}\right)$.

The additional scalars $\varphi$ (dilaton), $\tilde{\phi}$ (axion) and $\zeta^{I}, \tilde{\zeta}_{I}, I=0, \ldots h^{2,1}$ (R-R scalars) extend this SK manifold either to a quaternion-Kähler manifold (QK manifold) or to a para-quaternion-Kähler manifold (PQK manifold). Which case is realized depends on the signs of the kinetic terms of the R-R scalars. The HM Lagrangian takes the form

$$
\begin{aligned}
L_{\mathrm{HM}}^{\mathrm{IIA}}= & -\tilde{G}_{\alpha \bar{\beta}} d z^{\alpha} \wedge \star d \bar{z}^{\bar{\beta}}-\frac{1}{4} d \varphi \wedge \star d \varphi \\
& -e^{-2 \varphi}\left[d \tilde{\phi}+\frac{1}{2}\left(\zeta^{I} d \tilde{\zeta}_{I}-\tilde{\zeta}_{I} d \zeta^{I}\right)\right] \wedge \star\left[d \tilde{\phi}+\frac{1}{2}\left(\zeta^{I} d \tilde{\zeta}_{I}-\tilde{\zeta}_{I} d \zeta^{I}\right)\right] \\
& -\frac{\lambda}{2} e^{-\varphi}\left[\mathcal{I}_{I J} d \zeta^{I} \wedge \star d \zeta^{J}+\mathcal{I}^{I J}\left(d \tilde{\zeta}_{I}+\mathcal{R}_{I K} d \zeta^{K}\right) \wedge \star\left(d \tilde{\zeta}_{I}+\mathcal{R}_{I K} d \zeta^{K}\right)\right],
\end{aligned}
$$

where $\lambda=-1$ for type-IIA $(1,9)[16]$ and type-IIA $(0,10)[18]$ and $\lambda=1$ for type-IIA $(1,9)$ and type-IIA $_{(2,8)}$. The coupling matrices $\mathcal{I}_{I J}$ and $\mathcal{R}_{I J}$ depend on the complex scalars $z^{\alpha}$ and are determined by the prepotential $\mathcal{F}\left(z^{\alpha}\right)$ by the same formulae as vector field couplings for vector multiplets. This reflects that the HM manifolds resulting from CY3 compactifications are not generic, but of a special type, which can be obtained from a SK manifolds by the c-map. In the case at hand the SK manifold is the complex structure moduli space, and the c-map is either the standard c-map or the temporal c-map. These c-maps can be defined using the reduction of a Lorentz signature VM Lagrangian to three dimensions either of space or over time. That the same types of HM manifolds occur in CY3 compactifications 


\begin{tabular}{|l|l|l|}
\hline $10 \mathrm{~d}$ & $4 \mathrm{~d}$ & \\
\hline$C$ & $\mathrm{c}$ & scalar \\
\hline$C_{M N}$ & $C_{\mu \nu} \sim a$ & scalar \\
& $C_{m n} \sim u^{A}$ & $h^{1,1}$ scalars \\
\hline$C_{M N P Q}$ & $C_{\mu \nu m n} \sim v^{A}$ & $h^{1,1}$ scalars \\
& $C_{\mu m n p} \sim \mathcal{A}_{\mu}^{0}, \mathcal{A}_{\mu}^{A}$ & $1+h^{2,1}$ vector fields \\
\hline
\end{tabular}

Table 11. Massless fields in type-IIB Calabi-Yau compactifications, R-R sector.

is no coincidence, but related to the fact that T-duality of type-II CY3 compactifications exchanges VMs and HMs, as we will see later. The HM geometry is QK for type-IIA $(1,9)$ and type-IIA $_{(0,10)}$ (and positive definite, since in our convention $\mathcal{I}_{I J}$ is negative definite), and PQK for type-IIA $(1,9)$ and type-IIA $(2,8)$. Note that in all cases the submanifold parametrized by the scalars $z^{\alpha}$ is an SK manifold.

\subsection{Type-IIB Calabi-Yau compactifications}

We now turn to type-IIB compactifications. The NS-NS sector is the same as for type-IIA. In the R-R sector the zero form $C_{0}$ gives rises to a scalar $c$. The two-form $C_{2}$ gives rise to a two-form $C_{\mu \nu}$ which we dualize into a scalar $a$, and to $h^{1,1}$ scalars $u^{A}, A=1, \ldots, h^{1,1}$. Taking into account the self-duality of the five-form $G_{5}$, the four-form $C_{4}$ gives rise to $h^{1,1}$ two-forms $C_{\mu \nu}^{A}$ which we dualize to scalars $v^{A}$, and $1+h^{2,1}$ vectors $\mathcal{A}_{\mu}^{0}$ and $\mathcal{A}_{\mu}^{\alpha}$. The first vector is associated to the harmonic $(3,0)$-form of the CY3, while the other vectors correspond to the harmonic (2,1)-forms. See table 11 for a summary.

Together with the NS-NS fields, these fields are the bosonic content of the supergravity multiplet, $\left(g_{\mu \nu}, \mathcal{A}_{\mu}^{0}\right)$, of $h^{2,1}$ vector multiplets $\left(z^{\alpha}, A_{\mu}^{\alpha}\right)$, and of $h^{1,1}+1$ hypermultiplets $\left(y^{A}, x^{A}, \varphi, \tilde{\phi}, u^{A}, v^{A}, c, a\right)$. The vector fields can be rearranged into linear combinations $A_{\mu}^{I}$ with field strength $F_{\mu \nu}^{I}$ which together with the dual field strength $G_{I \mid \mu \nu}$ form a symplectic vector. The relative signs between the kinetic terms are determined by those between the ten-dimensional fields and are listed in table 12.

While one can perform the reduction of type-IIB theories explicitly, see for example [17] for $\operatorname{IIB}_{(1,9)}$, we can infer the result by using mirror symmetry and tracing sign flips. As is well known, type $\operatorname{IIA}_{(1,9)}$ compactified on a CY3 with Hodge numbers $\left(h^{1,1}, h^{2,1}\right)$ is equivalent to $\operatorname{IIB}_{(1,9)}$ compactified on the mirror CY3 with Hodge numbers $\left(h^{\prime 1,1}=h^{2,1}, h^{\prime 2,1}=h^{1,1}\right)$. Both theories have $n_{V}=h^{1,1}=h^{\prime 2,1}$ vector multiplets and $n_{H}=h^{2,1}+1=h^{\prime 1,1}+1$ hypermultiplets. In IIA compactifications complex structure module sit in hypermultiplets and Kähler moduli in vector multiplets while in IIB compactifications it is the other way round. We would like to compactify the IIB theory on the same CY3 as the IIA theory, but this is the same as compactifying the IIA theory on the mirror. The resulting theory has $n_{V}=h^{2,1}$ vector multiplets and $n_{H}=h^{1,1}+1$ hypermultiplets, and the action can be brought to our preferred standard form of a vector and hypermultiplet action. To adapt results from type-IIB to type-IIB* and type-IIB', we then only have to trace the effect of the ten-dimensional sign flips. 


\begin{tabular}{|l|l||l|l|l|l|l|l|l|l||l|}
\hline & $z^{\alpha}$ & $y^{A}$ & $x^{A}$ & $\varphi$ & $\tilde{\phi}$ & $c$ & $a$ & $u^{A}$ & $v_{A}$ & $A_{\mu}^{I}$ \\
\hline IIB & + & + & + & + & + & + & + & + & + & + \\
IIB $^{*}$ & + & + & + & + & + & - & - & - & - & - \\
IIB $^{\prime}$ & + & + & - & + & - & - & + & + & - & - \\
\hline
\end{tabular}

Table 12. Signs of the kinetic terms for scalar and vector fields resulting from type-IIB CY3 compactifications. A + indicates a standard kinetic term. The fields $z^{\alpha}$ are the vector multiplet scalars.

As a result, the bosonic Lagrangian for the supergravity multiplet and the $n_{V}=h_{2,1}$ vector multiplets takes the form

$$
L_{G+\mathrm{VM}}^{\mathrm{IIB} / \mathrm{IIB}^{*} / \mathrm{IIB}^{\prime}}=\frac{1}{2} \star R_{4}-\bar{g}_{\alpha \bar{\beta}}(z, \bar{z}) d z^{\alpha} \wedge \star d \bar{z}^{\bar{\beta}}-\frac{\lambda}{4} \mathcal{I}_{I J} F^{I} \wedge \star F^{J}+\frac{1}{4} \mathcal{R}_{I J} F^{I} \wedge F^{J},
$$

where $\lambda=-1$ for IIB, and $\lambda=1$ for IIB* and IIB'. The geometry is SK, with the two cases distinguished by an overall sign flip of the gauge fields.

In the hypermultiplet sector we can rearrange the scalars into linear combinations $\zeta^{I} \sim c, v^{A}$ and $\tilde{\zeta}_{I} \sim a, u^{A}$. The IIB HM Lagrangians take the form

$$
\begin{aligned}
L_{\mathrm{HM}}^{\mathrm{IIB} / \mathrm{IIB}^{*} / \mathrm{IIB}^{\prime}}= & -\tilde{G}_{A \bar{B}} d z^{A} \wedge \star d \bar{z}^{\bar{B}}-\frac{1}{4} d \varphi \wedge \star d \varphi \\
& +\epsilon_{1} e^{-2 \varphi}\left[d \tilde{\phi}+\frac{1}{2}\left(\zeta^{I} d \tilde{\zeta}_{I}-\tilde{\zeta}_{I} d \zeta^{I}\right)\right] \wedge \star\left[d \tilde{\phi}+\frac{1}{2}\left(\zeta^{I} d \tilde{\zeta}_{I}-\tilde{\zeta}_{I} d \zeta^{I}\right)\right] \\
& -\frac{\epsilon_{2}}{2} e^{-\varphi}\left[\mathcal{I}_{I J} d \zeta^{I} \wedge \star d \zeta^{J}-\epsilon_{1} \mathcal{I}^{I J}\left(d \tilde{\zeta}_{I}+\mathcal{R}_{I K} d \zeta^{K}\right) \wedge \star\left(d \tilde{\zeta}_{I}+\mathcal{R}_{I K} d \zeta^{K}\right)\right] .
\end{aligned}
$$

For type-IIB the scalars $z^{A}=y^{A}+i x^{A}$ are complex and parametrize an SK submanifold. The parameters $\epsilon_{1}, \epsilon_{2}$ take the values $\left(\epsilon_{1}, \epsilon_{2}\right)=(-1,-1)$, the HM manifold is positive definite and QK. For type-IIB* the signs of all R-R scalars are flipped, and $\left(\epsilon_{1}, \epsilon_{2}\right)=(-1,1)$. The scalars $z^{A}$ are again complex and span an SK submanifold, and the HM manifold is PQK. When going from IIB* to IIB', the signs of $x^{A}, \tilde{\phi}$ and of $\tilde{\zeta}_{I} \sim a, u^{A}$ are flipped. The scalars $y^{A}$ and $x^{A}$ now combine into para-complex scalars $z^{A}=y^{A}+e x^{A}$ which parametrize an SPK submanifold. We now have $\left(\epsilon_{1}, \epsilon_{2}\right)=(1,1)$. This is again a PQK manifold, but with a PSK submanifold instead of an SK manifold. Thus the S-duality relating type-IIB* to type-IIB' changes the HM manifolds in a significant way, while keeping it consistent with the same supersymmetry algebra.

Comparing type-IIA with type-IIB compactifications on the same CY3, we see that, loosely speaking, vector and hypermultiplet get exchanged. A type-IIA compactification has $n_{V}=h^{1,1}$ vector and $n_{H}=h^{2,1}+1$ hypermultiplets, while a type-IIB compactification has $n_{V}^{\prime}=h^{2,1}$ vector and $n_{H}^{\prime}=h^{1,1}+1$ hypermultiplets. As indicated by the Hodge numbers, complex structure moduli of the CY3 metric end up in HMs for type-IIA and in VMs for type-IIB, while (para)-complexified Kähler moduli end up in VMs for type-IIB and in HMs for type-IIA. In both cases there is a universal HM which contains the dilaton, axion and two R-R fields. Moreover, in both cases all model dependence, that is the dependence on the 
choice of the CY3, is encoded in two functions, the holomorphic prepotential of the complex structure moduli space and the (para)-holomorphic prepotential of the (para)complexified Kähler moduli space. ${ }^{9}$ The complex structure moduli space is of course always complex, but the Kähler moduli space becomes para-complex for type-IIA $(0,10)$ and type-IIA $(2,8)$ as well as type-IIB' ${ }_{(1,9)}$ due to the sign flip of the Kalb-Ramond field. The HM manifolds are completely determined by their distinguished $\mathrm{S}(\mathrm{P}) \mathrm{K}$ submanifold through a c-map. This structure is consistent with certain pairs of compactifications being 'on the same moduli' after compactification to three dimensions. As a result, type-II compactifications are mutually related by T-dualities transverse to the CY3. This will be studied in detail in the next section.

\section{$5 \quad$ T-duality}

We can now combine the results about c-maps with those about CY3 compactifications to determine how the four-dimensional theories resulting from type-II CY3 compactifications are related by T-duality. Type-IIA $(1,9)$ string theory compactified on a circle of radius $R$, measured in string units $\sqrt{\alpha^{\prime}}$, is equivalent to type-IIB $(1,9)$ compactified on a circle of radius $1 / R[45,46]$. Moreover, type-IIB $(1,9)$ string theory on ten-dimensional Minkowski space can be obtained as an alternative decompactification limit $R \rightarrow 0$ of the circle compactified type-IIA $(1,9)$ theory, with winding modes playing the roles of momentum modes, and vice versa. This is what is meant when saying that the uncompactified theories 'are T-dual to each other.' T-duality extends to backgrounds which include a compact factor transverse to the circle. In particular type-IIA $(1,9)$ compactified on $X \times S_{R}^{1}$, is equivalent to type-IIB $(1,9)$ compactified on $X \times S_{1 / R}^{1}$, where $X$ is the same CY3. By taking the alternative decompactification limit $R \rightarrow \infty$, one can map the four-dimensional effective field theories for type-IIA $(1,9)$ and type-IIB $(1,9)$, compactified on the same CY3 $X$, to one another, and the relation between the respective vector and hypermultiplet sectors is given by the c-map and its inverse [28]. Timelike T-dualities and mixed T-dualities which combine spacelike/timelike reduction with timelike/spacelike oxidation, together with S-duality, relate all ten-dimensional type-II theories to one another $[1,2]$. In this section we extend these T-dualities to CY3 compactifications. We remark that it is straightforward though somewhat tedious to work out the explicit relations between the fields of two T-dual four-dimensional effective field theories. T-duality operates naturally in the string frame, and therefore we would need to convert our actions from the Einstein frame to the string frame, perform the reductions of T-dual theories over circles of radii $R$ and $1 / R$, and then read off the relations between the fields. While the explicit map between fields is needed for some applications, in particular for mapping solutions from one theory to solutions of a T-dual theory, we will only be interested in how the various type-II CY3 compactifications are related to each other by T-duality and S-duality. For this it is sufficient to match the hypermultiplet manifolds that we get after reduction to three dimensions, as this show that

\footnotetext{
${ }^{9}$ To see that the two prepotentials are on the same footing one must go beyond a simple dimensional reduction and include the $\alpha^{\prime}$-corrections to the Kähler moduli space. We refer to [44] for a review of string theory on Calabi-Yau manifolds.
} 
both four-dimensional theories reduce to the same three-dimensional theory. All that we need for this comparison was worked out in section 2. Explicit maps between the fields will be given in a future publication where we will study the action of T-duality on solutions of the four-dimensional effective field theories.

\subsection{Signature $(1,3)$ and spacelike/timelike T-duality}

To start exploring the web of relations between four-dimensional theories we begin with the CY3 compactification of the type-IIA $(1,9)$ theory.

- Type-IIA $(1,9)$ string theory on a Calabi-Yau threefold has $n_{V}=h^{1,1}$ vector and $n_{H}=h^{2,1}$ hypermultiplets. It realizes the standard $\mathcal{N}=2$ algebra with R-symmetry $\mathrm{U}(2) \cong \mathrm{U}(1) \times \mathrm{SU}(2)$ and the scalar manifold has the form

$$
\mathcal{M}^{\mathrm{IIA}}=\mathcal{M}_{2 h^{1,1}}^{\mathrm{SK}} \times \tilde{\mathcal{N}}_{4 h^{2,1}+4}^{\mathrm{QK}} .
$$

Upon spacelike reduction the scalar manifold becomes the product of two QK manifolds

$$
\mathcal{M}^{(1,2)}=\mathcal{N}_{4 h^{1,1}+4}^{\mathrm{QK}} \times \tilde{\mathcal{N}}_{4 h^{2,1}+4}^{\mathrm{QK}}
$$

If one swaps the roles of the two factors and lifts back over space, one obtains

$$
\mathcal{M}^{\mathrm{IIB}}=\mathcal{N}_{4 h^{1,1}+4}^{\mathrm{QK}} \times \tilde{\mathcal{M}}_{2 h^{2,1}}^{\mathrm{SK}}
$$

as required for a Calabi-Yau compactification of type-IIB string theory. This is the standard, spatial T-duality between type-IIA and type-IIB, extended to their CalabiYau compactifications. It employs the standard, spatial c-map in both directions.

- If we start again with type-IIA, but perform a timelike reduction, we obtain a theory in signature $(0,3)$ with scalar target

$$
\mathcal{M}^{(0,3)}=\mathcal{N}_{4 h^{1,1}+4}^{\mathrm{PQK}} \times \tilde{\mathcal{N}}_{4 h^{2,1}+4}^{\mathrm{QK}},
$$

where the first factor is now PQK rather than QK. Swapping the two factors and lifting back over time we obtain a scalar manifold of the form

$$
\mathcal{M}^{\mathrm{IIB}}=\mathcal{N}_{4 h^{1,1}+4}^{\mathrm{PQK}} \times \tilde{\mathcal{M}}_{2 h^{2,1}}^{\mathrm{SK}} .
$$

Note that after the oxidation we have flipped gauge field terms (recorded as SK_) since we need such a sign in order to obtain a QK manifold by timelike reduction. The resulting four-dimensional theory realizes the twisted Lorentz signature algebra with R-symmetry $\mathrm{U}(1,1) \cong \mathrm{U}(1) \times \mathrm{SU}(1,1)$. Thus we obtain the timelike T-duality between type-IIA and type-IIB*, extended to their Calabi-Yau compactifications. It employs the temporal c-map for reduction and the twisted temporal c-map for oxidation. 
- If we start with type-IIA* the initial scalar manifold is

$$
\mathcal{M}^{\mathrm{IIA}}=\mathcal{M}_{2 h^{1,1}}^{\mathrm{SK}} \times \tilde{\mathcal{N}}_{4 h^{2,1}+4}^{\mathrm{PQK}_{\mathrm{SK}}}
$$

Upon space-like reduction this becomes

$$
\mathcal{M}^{(1,2)}=\mathcal{N}_{4 h^{1,1}+4}^{\mathrm{PQK}} \times \tilde{\mathcal{N}}_{4 h^{2,1}+4}^{\mathrm{PQK}},
$$

which lifts back to

$$
\mathcal{M}^{\mathrm{IIB}}=\mathcal{N}_{4 h^{1,1}+4}^{\mathrm{PQK}} \times \tilde{\mathcal{M}}_{2 h^{2,1}}^{\mathrm{SK}} .
$$

This realizes the spacelike T-duality between type-IIA* and type-IIB*, extended to their Calabi-Yau compactifications. Here we employ the twisted spatial c-map in both directions.

- If we start with type-IIA* and reduce over time we obtain instead

$$
\mathcal{M}^{(0,3)}=\mathcal{N}_{4 h^{1,1}+4}^{\mathrm{QK}} \times \tilde{\mathcal{N}}_{4 h^{2,1}+4}^{\mathrm{PQK}},
$$

which lifts back to

$$
\mathcal{M}^{\mathrm{IIB}}=\mathcal{N}_{4 h^{1,1}+4}^{\mathrm{QK}} \times \tilde{\mathcal{M}}_{2 h^{2,1}}^{\mathrm{SK}},
$$

and we realize the timelike T-duality between Calabi-Yau compactifications of type IIA $^{*}$ and type IIB. Here we use the twisted temporal c-map for reduction and the temporal c-map for oxidation.

The relations between the four-dimensional theories are summarized by the lower face of the cubic diagram in figure 2 .

\subsection{Mixed T-dualities and signature change}

Let us now mix spacelike/timelike reduction with timelike/spacelike oxidation in order to relate four-dimensional theories across signatures. If we start with the CY3 compactification of type-IIA $(0,10)$ we have a scalar manifold which is the product of an SPK and a QK manifold:

$$
\mathcal{M}^{\mathrm{IIA},(0,4)}=\mathcal{M}_{2 h^{1,1}}^{\mathrm{SPK}} \times \tilde{\mathcal{N}}_{4 h^{2,1}+4}^{\mathrm{QK}} .
$$

Upon spacelike reduction to signature $(0,3)$, the scalar manifold becomes

$$
\mathcal{M}^{(0,3)}=\mathcal{N}_{4 h^{1,1}+4}^{\mathrm{PQK}} \times \tilde{\mathcal{N}}_{4 h^{2,1}+4}^{\mathrm{QK}},
$$

where the first PQK manifold has an SPK base. This step involves the Euclidean c-map. We now need to identify a IIB theory that gives rise to the same scalar manifold upon timelike reduction. To obtain the QK manifold $\tilde{\mathcal{N}}_{4 h^{2,1}+4}^{\mathrm{QK}}$ by timelike reduction we need to start with vector multiplets which have SK geometry and a sign flip between scalar and vector term, denoted SK_. This could be either the CY3 compactification of IIB* or IIB'. The map $\tilde{\mathcal{M}}_{2 h^{2,1}}^{\mathrm{SK}} \rightarrow \tilde{\mathcal{N}}_{4 h^{2,1}+4}^{\mathrm{QK}}$ is the twisted version of the temporal c-map. The HM manifold of the 


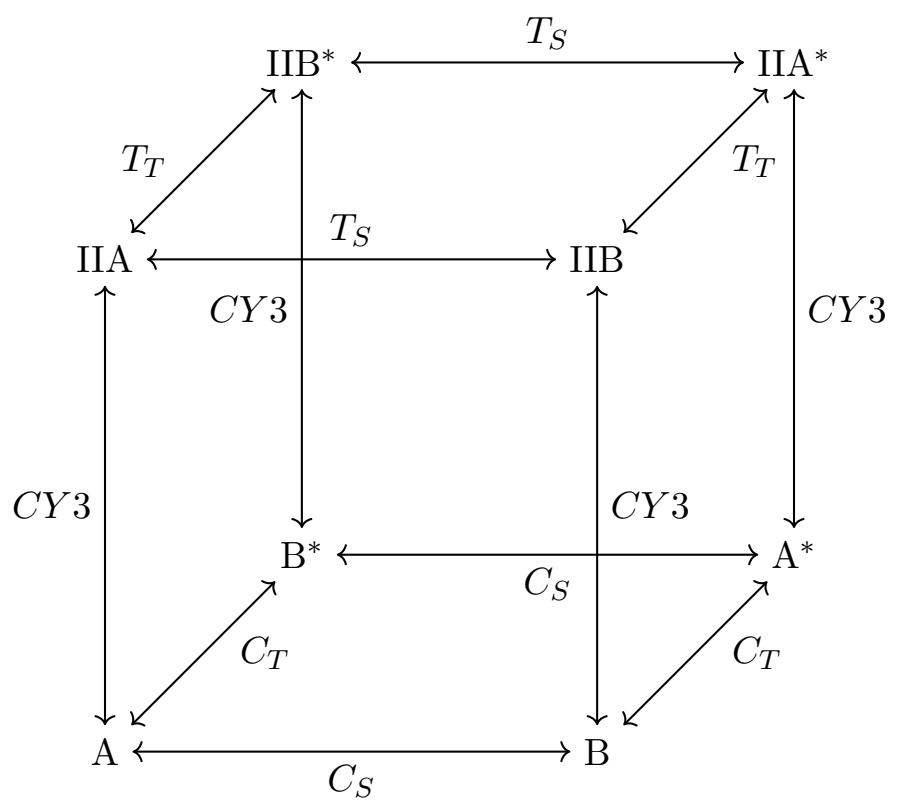

Figure 2. The spacelike and timelike T-dualities $T_{S}, T_{T}$ between the four type-II string theories in ten-dimensional Minkwoski space induce relations between the four-dimensional supergravity theories, denoted A, B, A*, B* obtained by compatification on the same Calabi-Yau threefold. The number $n_{V}$ of vector multiplets and $n_{H}$ of hypermultiplets is related to the Hodge number of the Calabi-Yau threefold by $\left(n_{V}, n_{H}\right)=(m, n)=\left(h_{1,1}, h_{2,1}+1\right)$ for type-A and $\left(n_{V}, n_{H}\right)=\left(m^{\prime}, n^{\prime}\right)=\left(h_{2,1}, h_{1,1}+1\right)$ for type-B. The theories denoted $A^{*}, B^{*}$ have the same structure, but a modified supersymmetry algebra with a non-compact R-symmetry group which results in sign flips in the Lagrangian and modifications of the scalar geometry. The maps relating the four-dimensional theories are denoted $C_{S}, C_{T}$, depending on whether they use a spacelike or timelike reduction and oxidation.

partner theory must match $\mathcal{N}_{4 h^{1,1}+4}^{\mathrm{PQK}_{\mathrm{SPK}}}$, that is, it must be a PQK manifold with an SPK base. Therefore we need to choose IIB', which has a scalar manifold of the type

$$
\mathcal{M}^{\mathrm{IIB}^{\prime}}=\mathcal{N}_{4 h^{2,1}+4}^{\mathrm{PQK}} \times \tilde{\mathcal{M}}_{2 h^{1,1}}^{\mathrm{SK}} .
$$

This shows the existence of a mixed T-duality relating the CY3 compactifications of type$\operatorname{IIA}_{(0,10)}$ and type-IIB' $(1,9)$, which uses the Euclidean c-map for reduction and the twisted temporal c-map for oxidation.

If we reduce the IIB' theory over space, the resulting scalar manifold is

$$
\mathcal{M}^{(1,2)}=\mathcal{N}_{4 h^{2,1}}^{\mathrm{PQK}} \times \tilde{\mathcal{N}}_{4 h^{1,1}+4}^{\mathrm{PQK}}
$$

where $\tilde{\mathcal{M}}_{2 h^{1,1}}^{\mathrm{SK}} \rightarrow \tilde{\mathcal{N}}_{4 h^{1,1}+4}^{\mathrm{PQK}_{\mathrm{SK}}}$ is the twisted version of the spatial c-map.

By lifting this back over time we obtain the scalar manifold of the CY3 compactification of $\operatorname{IIA}_{(2,8)}$,

$$
\mathcal{M}^{\mathrm{IIA},(2,2)}=\mathcal{M}_{2 h^{1,1}}^{\mathrm{SPK}} \times \tilde{\mathcal{N}}_{4 h^{2,1}+4}^{\mathrm{PQK}}
$$

This step involves the inverse of the neutral c-map which maps SPK to PQK through a timelike reduction. In summary we have shown the existence of a mixed T-duality relating 


\begin{tabular}{|l|l||l|l||l|l|}
\hline IIA in 4d & Scalar mfd. & 3d sig. & Scalar mfd. & IIB in 4d & Scalar mfd. \\
\hline $\mathrm{IIA}_{(0,4)}$ & SPK $\times \mathrm{QK}$ & $(0,3)$ & $\mathrm{PQK}_{\mathrm{SPK}} \times \mathrm{QK}$ & $\mathrm{IIB}_{(1,3)}$ & $\mathrm{PQK}_{\mathrm{SPK}} \times \mathrm{SK}_{-}$ \\
$\mathrm{IIA}_{(1,3)}$ & $\mathrm{SK}_{+} \times \mathrm{QK}$ & $(0,3)$ & $\mathrm{PQK}_{\mathrm{SK}} \times \mathrm{QK}$ & $\mathrm{IIB}_{(1,3)}^{*}$ & $\mathrm{PQK}_{\mathrm{SK}} \times \mathrm{SK}_{-}$ \\
$\mathrm{IIA}_{(1,3)}^{*}$ & $\mathrm{SK}_{-} \times \mathrm{PQK}_{\mathrm{SK}}$ & $(0,3)$ & $\mathrm{QK} \times \mathrm{PQK}_{\mathrm{SK}}$ & $\mathrm{IIB}_{(1,3)}$ & $\mathrm{QK} \times \mathrm{SK}_{+}$ \\
$\mathrm{IIA}_{(1,3)}$ & $\mathrm{SK}_{+} \times \mathrm{QK}$ & $(1,2)$ & $\mathrm{QK} \times \mathrm{QK}$ & $\mathrm{IIB}_{(1,3)}$ & $\mathrm{QK} \times \mathrm{SK}_{+}$ \\
$\mathrm{IIA}_{(1,3)}^{*}$ & $\mathrm{SK}_{-} \times \mathrm{PQK}_{\mathrm{SK}}$ & $(1,2)$ & $\mathrm{PQK}_{\mathrm{SK}} \times \mathrm{PQK}_{\mathrm{SK}}$ & $\mathrm{IIB}_{(1,3)}^{*}$ & $\mathrm{PQK}_{\mathrm{SK}} \times \mathrm{SK}_{-}$ \\
$\mathrm{IIA}_{(2,2)}$ & SPK $\times \mathrm{PQK}_{\mathrm{SK}}$ & $(1,2)$ & $\mathrm{PQK}_{\mathrm{SPK}} \times \mathrm{PQK}_{\mathrm{SK}}$ & $\mathrm{IIB}_{(1,3)}$ & $\mathrm{PQK}_{\mathrm{SPK}} \times \mathrm{SK}_{-}$ \\
\hline
\end{tabular}

Table 13. Summary of T-dualities between type-IIA and type-IIB Calabi-Yau compactifications for all (inequivalent) four-dimensional signatures. In the middle we specify the three-dimensional theory to which the T-dual four-dimensional theories reduce.

the CY3 compactifications of type-IIB' ${ }_{(1,9)}$ and type-IIA $(2,8)$ which uses the twisted spatial c-map for reduction and the neutral c-map for oxidation. We summarize the six T-dualities which relate type-IIA and type-IIB theories in four dimensions in table 13. Note that under T-duality the compactifications organise into two orbits: the orbit of 'pure' T-dualities relating IIA/IIA*/IIB/IIB* in signature $(1,3)$, and the orbit of mixed T-dualities relating type-IIA theories in signatures $(0,4)$ and $(2,2)$ to the type-IIB' theory in signatures $(1,3)$. In order connect these two orbits to one another we would need to use the duality between IIB* and IIB', which is an S-duality. Since CY3 backgrounds only preserve four-dimensional $\mathcal{N}=2$ supersymmetry, there is not good reason to expect that S-duality is valid, and therefore we should expect that there are two distinct classes of compactifications. The relation of the backgrounds within each orbit relies on T-duality for backgrounds of the form CY3 $\times S^{1}$ which is an established perturbative symmetry of string theory. Note however, that there are special, non-generic $\mathcal{N}=2$ compactifications which are ' $\mathcal{N}=4$-like' and exhibit S-duality. For this class all type-II CY3 compactifications should form a single orbit at the non-perturbative level by combining pure T-dualities, mixed T-dualities and S-duality. Note that for S-duality to work, there has to exist, for the HM manifolds of these special models, an isomorphism of PQK manifolds, which replaces an SK base with an SPK base. Such an isomorphism can only exist in special case when the structure of the prepotential for the $\mathrm{S}(\mathrm{P}) \mathrm{K}$ base is very simple.

Let us finally point out that considering the other ten-dimensional signatures will add nothing new. The type-II $\mathrm{I}_{(5,5)}$ theories cannot be compactified on CY3 folds, and the theories $\operatorname{IIA}_{(10,0)}, \operatorname{II}_{(9,1)}, \operatorname{IIA}_{(8,2)}$ are related to those we have considered by an overall sign change of the metric, which maps $(t, s) \rightarrow(s, t)$. Theories related in this way have been shown to be equivalent [2]. The type-IIA $(4,6)$ and type-IIA $(6,4)$ reduce to theories in signature $(4,0)$ and $(0,4)$ which are equivalent to those we have considered. From the higher-dimensional point of view the chain of mixed T-dualities is projected onto the chain we have described. Since the four-dimensional theory in signature $(0,4)$ is unique, the difference between type-IIA $_{(0,10)}$ and type-IIA $(4,6)$ is lost from the four-dimensional perspective. Similarly the type- $\operatorname{IIB}_{(3,7)}$ and type- $\operatorname{IIB}_{(7,3)}$ reduce to theories in signatures $(3,1)$ of $(1,3)$, which are of the type-IIB' type, and the distinction between type-IIB' ${ }_{(3,7)}$ and type-IIB' ${ }_{(1,9)}$ is lost from 
the four-dimensional perspective. ${ }^{10}$ We have already pointed out that in order to relate such a theory to type-IIB*, and thus to the other T-duality orbit, we need to use S-duality, which can only be expected to be a symmetry for non-generic $\mathcal{N}=2$ compactifications. Signatures $(3,7)$ and $(7,3)$ have a unique theory of type-IIB', but they can be related through mixed T-dualities to signatures $(1,9)$ and $(9,1)$ where by using S-duality they can be related to type-IIB* and from there by T-dualities to type IIA/IIA* and type-IIB. Thus for $\mathcal{N}=2$ compactifications which preserve S-duality, we can connect the CY3 compactifications of all type-II theories in signatures $(0,10), \ldots(4,6)$ to one another (and the same applies to signature obtained by an overall sign flip). For generic $\mathcal{N}=2$ we have to expect two disjoint T-duality orbits.

\section{Outlook}

In this paper we have obtained the CY3 compactifications of all ten-dimensional type-II string theories and analyzed how they are related to one another by T-duality and S-duality. At the level of symmetries and effective supergravity, we get a full and satisfactory picture which is consistent with the idea that exotic string theories and their compactifications fit into an extended string theory landscape and allow one to realize all maximally supergravity theories in all signatures as limits. Of course, admitting backgrounds with multiple time directions, as well as inverted kinetic terms in the effective action raises conceptual questions. Instead of repeating the arguments of $[1,2,4,5]$, let us ask what new insights and future directions result from our work.

Apart from symmetry considerations, a reason to consider the inclusion of exotic string theories into the string theory landscape is string universality, and the observation that bubbles of exotic spacetime signature can be generated [4]. One future direction is to explore in more detail whether and how dynamical signature change can be realized as a physical process in string theory. It would be interesting to relate this to the recent work [11-13] on complex spacetime metrics, which was in part motivated by earlier work [47] on topology change. Gravity with a dynamical signature has recently been discussed in [48] within the framework of Einstein-Cartan gravity.

To make a preliminary remark on this topic, we note that the T-duality orbit along which spacetime signature can change in type-II CY3 compactifications is connected to the orbit of the standard IIA/IIB compactifications and their IIA*/IIB* partners by S-duality, which we cannot expect to be valid in generic $\mathcal{N}=2$ compactifications. This suggests that in backgrounds with less than $\mathcal{N}=4$ supersymmetry, there is, generically (with the exception of special ' $\mathcal{N}=4$ like' backgrounds), a separation between a phase with Lorentzian string worldsheets and fixed Lorentzian spacetime signature, and a phase with Euclidean string worldsheets and arbitrary spacetime signature. ${ }^{11}$ Thus signature change

\footnotetext{
${ }^{10}$ The actions of $\operatorname{IIB}_{(1,9) /(9,1)}$ and IIB'$_{(3,7) /(7,3)}$ differ by an overall sign flip of the R-R fields [2, 4]. Upon compactification on a CY3, this results in a flip of the parameter $\epsilon_{2}$ in the hypermultiplet sector, but the resulting hypermultiplet manifolds are isometric.

${ }^{11}$ Lorentizan string worldsheets are also possible in neutral signature $(5,5)$, but this signature does not give rise to CY3 compactifications. Also, to connect it to the standard IIA/IIB theories, one needs to use S-duality.
} 
may only be relevant cosmologically if the universe goes through a phase of high unbroken supersymmetry. ${ }^{12}$

Based on the results of this paper, solutions of exotic string theories can now be explored systematically from a four-dimensional $\mathcal{N}=2$ perspective in addition to the ten-dimensional perspective. Future work will include how solutions transform under dualities, whether solutions of different theories can be connected to one another, including the question of dynamical signature change. Staying within the class of theories with Lorentzian string worldsheets, there are interesting questions regarding the relation between solutions in type-II and type-II*. The results of our paper imply that the dual pair of planar cosmological and black hole solutions described in [30,31] lifts to a 'dual pair' of solutions in type-IIA and type-IIA*. This raises the question whether these 'dual solutions' can be related by T-duality (which is not obvious). Both solutions have horizon thermodynamics that can be related to the same Euclidean thermal partition functions, and one can now ask whether the type-II embedding provides insights into the underlying microscopic physics. One could also study whether these solutions correspond to admissible saddle points, in the sense of [11-13], of the Euclidean path integral.

\section{Acknowledgments}

T.M. thanks Owen Vaughan for stimulating discussions at an early stage of this project. The work of Maxime Medevielle has been supported by an EPSRC DTP International Doctoral Scholarship (project number 2271092).

\section{A Calabi-Yau compactifications}

In this appendix we provide some more details on Calabi-Yau compactifications of type-IIA theories. Since this has been worked out in detail for $\operatorname{IIA}_{(1,9)}$ in $[16]$ and for $\operatorname{IIA}_{(0,10)}$ in [18] all we need in order to include the additional cases of $\operatorname{IIA}_{(1,9)}^{*}$ and $\operatorname{IIA}_{(2,8)}$, is to trace the ten-dimensional sign flips through the computation. The results from the reduction of the individual terms is taken from [18] whose conventions and notation we follow. We refer to $[22,49,50]$ for further background on CY3 compactifications and special geometry.

\section{A.1 Calabi-Yau threefolds}

A Calabi-Yau threefold (CY3) $X$ is a compact complex manifold of (complex) dimension three which admits a Ricci-flat Kähler metric. This condition is equivalent to the existence of a nowhere vanishing holomorphic $(3,0)$-form $\Omega$. We denote local real coordinates on $X$ by $y^{a}, a=1, \ldots, 6$ and introduce complex coordinates $\xi^{i}, i=1,2,3$

$$
\xi^{1}=\frac{1}{\sqrt{2}}\left(y^{1}+i y^{2}\right), \quad \xi^{2}=\frac{1}{\sqrt{2}}\left(y^{3}+i y^{4}\right), \quad \xi^{3}=\frac{1}{\sqrt{2}}\left(y^{5}+i y^{6}\right) .
$$

\footnotetext{
${ }^{12}$ Our universe could still be a brane world embedded into a higher-dimensional universe with multiple time directions, an option that has been explored in [5].
} 
The components of the Kähler metric in complex coordinates are $g_{i \bar{j}}$, and the volume form is

$$
\operatorname{vol}_{g}=\sqrt{g} d^{6} y=i \sqrt{g} d^{3} \xi d^{3} \bar{\xi} .
$$

Therefore the volume of $X$ is

$$
\mathcal{V}=\int_{X} \operatorname{vol}_{g}=\int_{X} \sqrt{g} d^{6} y=\int_{X} i \sqrt{g} d^{3} \xi d^{3} \bar{\xi} .
$$

The scalar product between differential $(p, q)$-form is

$$
\left(\omega_{(p, q)}, \eta_{(p, q)}\right)=\int_{X} \omega_{(p, q)} \wedge \star \eta_{(p, q)},
$$

where $\star$ is the Hodge operator with respect to the Kähler metric $g_{i \bar{j}}$. For reference we note that

$$
\star \rho_{(3,0)}=-i \rho_{(3,0)}, \quad \sigma_{(2,1)}=i \sigma_{(2,1)} .
$$

The massless spectrum of a CY3 compactification is determined by the harmonic $(p, q)$-forms. On a compact Kähler manifold, harmonic $(p, q)$-forms represent elements of the Dolbeault cohomology groups $H_{\bar{\partial}}^{p, q}(X, \mathbb{C})$. The Hodge numbers $h^{p, q}=\operatorname{dim} H_{\bar{\partial}}^{p, q}(X, \mathbb{C})$ of a CY3 are

$$
\begin{array}{ll}
h^{0,0}=h^{3,0}=h^{0,3}=h^{3,3}=1, & h^{1,0}=h^{0,1}=h^{3,2}=h^{2,3}=0, \\
h^{1,1}=h^{2,2} \geq 1, & h^{1,2}=h^{2,1} \geq 0 .
\end{array}
$$

We introduce a basis for harmonic forms representing Dolbeault cohomology classes:

$$
\begin{array}{rlrl}
V^{A} & =V_{i \bar{j}}^{A} d \xi^{i} \wedge d \bar{\xi}^{j}, & & A=1, \ldots, h_{1,1} \\
\Phi_{\alpha} & =\frac{1}{2} \Phi_{\alpha i j \bar{k}} d \xi^{i} \wedge d \xi^{j} \wedge d \bar{\xi}^{k}, & & \alpha=1, \ldots, h_{2,1} \\
\Omega & =\frac{1}{3 !} \Omega_{i j k} d \xi^{i} \wedge d \xi^{j} \wedge d \xi^{k} . &
\end{array}
$$

For the harmonic three-forms we also introduce a real basis $\alpha_{I}, \beta^{J}$, where $I=0, \ldots, h_{2,1}$, which is dual to a canonical basis of third homology group $H_{3}(X, \mathbb{Z})$ :

$$
\int_{A^{I}} \alpha_{J}=\int_{X} \alpha_{I} \wedge \beta^{J}=\delta_{I}^{J}=A_{I} \cdot B^{J} \quad \int_{B_{I}} \beta^{J}=\int_{X} \beta^{J} \wedge \alpha_{I}=-\delta_{I}^{J}=-B^{J} \cdot A_{I} .
$$

Here '.' denotes the intersection product of three-cycles. Observe that $H_{3}(X, \mathbb{Z})$ and $H^{3}(X, \mathbb{R})$ carry a natural symplectic structure.

Since $X$ is a Kähler manifold, once we have fixed a complex structure the metric is determined by the choice of a Kähler form. This is a harmonic (1,1)-form $J=M^{A} V^{A}$ which satisfies the positivity requirements

$$
\mathcal{K}=\int_{X} J \wedge J \wedge J=6 \mathcal{V}>0, \quad \int_{D^{A}} J \wedge J=\int_{X} V_{A} \wedge J \wedge J>0, \quad \int_{C_{A}} J=\int V^{A} \wedge J>0,
$$

where $C_{A}$ and $D^{A}$ are generators of $H_{2}(X, \mathbb{Z})$ and $H_{4}(X, \mathbb{Z})$ and $V_{A}, V^{A}$, ar the dual generators of $H^{4}(X, \mathbb{R})$ and $H^{2}(X, \mathbb{R})$. Thus the positivity conditions ensure that the volumes of $X$ as well as those of all surfaces and curves in $X$ are positive. The Kähler form is related to the metric by

$$
J=i g_{i j} d \xi^{i} \wedge d \bar{\xi}^{j}
$$


Since the metric $g_{i \bar{j}}$ is Kähler, its deformations split up into two types.

1. Deformations of the form $\delta g_{i \bar{j}}$ preserve the complex structure. When imposing in addition that Ricci flatness is preserved, the independent deformations (after taking into account reparametrizations) can be parametrized as

$$
i \delta g_{i \bar{j}}=\sum_{A=1}^{h^{1,1}} \delta M^{A} V^{A}
$$

where $M^{A}$ are real parameters and $V^{A}$ is our basis for the harmonic $(1,1)$-forms. Thus these deformations correspond to deformations of the Kähler form.

2. Deformations of the form $\delta g_{i j}, \delta g_{\overline{i j}}=\overline{\delta g_{i j}}$ change the complex structure. Inequivalent changes of the complex structure are parametrized by elements of $H^{1}(X, T X) \cong$ $H^{2,1}(X)$, where we used that the holomorphic top form can be used to identify vector-valued $(0,1)$-forms with $(2,1)$ forms:

$$
\phi_{i j \bar{k}}:=\Omega_{i j l} \psi_{\bar{k}}^{l}
$$

The inequivalent complex structure changing deformations of the metric can be parametrized using harmonic (2,1)-forms:

$$
\delta g_{\overline{i j}}=\sum_{\alpha=1}^{h^{2,1}} \delta z^{\alpha} b_{\alpha \overline{i j}}, \quad \text { where } \quad b_{\alpha}=\frac{1}{2} b_{\alpha \overline{i j}} d \bar{\xi}^{\bar{i}} \wedge d \bar{\xi}^{\bar{j}}=-\frac{i}{2} \frac{1}{\|\Omega\|^{2}} \bar{\Omega}_{\bar{i}}^{k l} \Phi_{\alpha k l \bar{j}} d \bar{\xi}^{\bar{i}} \wedge d \overline{\bar{\xi}}^{\bar{j}},
$$

where $z^{\alpha}$ are complex parameters, where

$$
\|\Omega\|^{2}=\frac{1}{3 !} \Omega_{i j k} \bar{\Omega}^{i j k}
$$

and where $\Phi_{\alpha}$ is our basis for the harmonic (2,1)-forms.

In CY3-compactifications the parameters $M^{A}, z^{\alpha}$ become scalar fields, which appear in the action through sigma models whose target spaces are the moduli spaces of Kähler structures and of complex structures, equipped with their natural metrics, about which we will report next. The so-called Weil-Peterson metric on the space of complex structures can be expressed through scalar products of harmonic forms as follows:

$$
g_{\alpha \bar{\beta}}=\frac{1}{\mathcal{V}}\left(b_{\alpha}, \bar{b}_{\bar{\beta}}\right)=\frac{1}{\|\Omega\|^{2} \mathcal{V}}\left(\Phi_{\alpha}, \bar{\Phi}_{\bar{\beta}}\right)=\frac{-i \int_{X} \Phi_{\alpha} \wedge \bar{\Phi}_{\bar{\beta}}}{i \int_{X} \Omega \wedge \bar{\Omega}} .
$$

The metric $g_{\alpha \bar{\beta}}$ is a Kähler metric, in fact a projective special Kähler metric of precisely the same type as appears for the target spaces of four-dimensional vector multiplets coupled to supergravity. To make this structure explicit, one notes that the complex structure of $X$ is encoded by the 'direction' of $H_{\bar{\partial}}^{3,0}(X, \mathbb{C})$ inside $H^{3}(X, \mathbb{C})$. To parametrize the choice of a complex structure one can use the periods of the holomorphic top-form $\Omega \in H_{\bar{\partial}}^{3,0}(X, \mathbb{C}) \subset H^{3}(X, \mathbb{C}):$

$$
X^{I}=\int_{A_{I}} \Omega, \quad F_{J}=\int_{B^{J}} \Omega .
$$


The top form can be expanded as

$$
\Omega=X^{I} \alpha_{I}-F_{I} \beta^{I} .
$$

The periods $X^{I}, F_{J}$ are not independent. Without loss of generality, we can assume that the periods $X^{I}$ are independent and parametrize the inequivalent choices of a holomorphic top-form $\Omega$ among the three-forms. Then the periods $F_{J}$ are, at least locally, functions of the coordinates $X^{I}$, and for a generic choice of a basis, they form the gradient $F_{J}=\partial F / \partial X^{J}$ of a function $F\left(X^{I}\right)$, the prepotential, which is holomorphic and homogeneous of degree two. ${ }^{13}$ Holomorphic top forms which differ by a complex scalar factor, $\Omega \rightarrow \lambda \Omega, \lambda \in \mathbb{C}^{*}$ define the same complex structure. Therefore the $X^{I}$ are projective coordinates on the space $\mathcal{M}_{2 h^{2,1}}$ of complex structures. So-called special coordinates on $\mathcal{M}_{2 h^{2,1}}$, which parametrize inequivalent complex structures, are provided by the ratios $z^{\alpha}=X^{\alpha} / X^{0}$. The space parametrized by the periods $X^{I}$ is the space of holomorphic top-forms, which forms, at least locally, a complex cone $\mathcal{C M}_{2 h^{2,1}+2}$ over the moduli space of complex structures. Here we see the same type of special geometry arising which also characterizes the scalar geometry of four-dimensional vector multiplets. $\mathcal{C M}_{2 h^{2,1}+2}$ is a conical affine special Kähler (CASK) manifold, while $\mathcal{M}_{2 h^{2,1}}$ is the associated projective special Kähler manifolds. The special Kähler metrics on both spaces can be expressed using the prepotential $F\left(X^{I}\right)=\left(X^{0}\right)^{2} \mathcal{F}\left(z^{A}\right)$. The associated Kähler potentials $K_{\mathrm{CASK}}, K$ and metrics $N_{I J}, g_{\alpha \beta}$ are

$$
\begin{aligned}
& K_{\mathrm{CASK}}=i\left(X^{I} \bar{F}_{I}-F_{I} \bar{X}^{I}\right) \quad \Rightarrow N_{I J}=\frac{\partial^{2} K_{\mathrm{CASK}}}{\partial X^{I} \partial \overline{X^{J}}}=2 \operatorname{Im} F_{I J}, \\
& K=-\log \left(-i\left(X^{I} \bar{F}_{I}-F_{I} \bar{X}^{I}\right)\right) \Rightarrow g_{\alpha \bar{\beta}}=\frac{\partial^{2} K}{\partial z^{\alpha} \partial \bar{z}^{\bar{\beta}}} .
\end{aligned}
$$

Note that since $F\left(X^{I}\right)$ is homogeneous of degree two, its derivatives $F_{I}, F_{I J}, \ldots$ are homogeneous of degrees one, zero, .... This allows one to rewrite formulas in various ways, for example $K=-\log \left(\bar{X}^{I} N_{I J} X^{J}\right)$.

These are generic expressions for special Kähler geometry, which are valid without any reference to CY3-folds. In CY3 compactifications the special geometry data can be expressed in terms of CY3 data. In particular:

$$
(\Omega, \bar{\Omega})=i \int_{X} \Omega \wedge \bar{\Omega}=\|\Omega\|^{2} \mathcal{V}=-i\left(X^{I} \bar{F}_{i}-F_{I} \bar{X}^{I}\right)=-N_{I J} \bar{X}^{I} X^{J}
$$

where $N_{I J}$ is the CASK metric on $\mathcal{C M}_{2 h^{1,2}+2}$. The Kähler potential can be expressed in terms of the top form:

$$
K=-\log (\Omega, \bar{\Omega})=-\log \left(i \int_{X} \Omega \wedge \bar{\Omega}\right) \Rightarrow g_{\alpha \bar{\beta}}=\frac{\partial^{2}}{\partial z^{\alpha} \partial \bar{z}^{\bar{\beta}}}\left(-\log \left(i \int_{X} \Omega \wedge \bar{\Omega}\right)\right) .
$$

Let us now consider deformations of the Kähler form. Following [16], we define the following integrals

$$
\begin{aligned}
\mathcal{K} & =\int_{C Y_{3}} J \wedge J \wedge J, & \mathcal{K}_{A} & =\int_{C Y_{3}} V^{A} \wedge J \wedge J, \\
\mathcal{K}_{A B} & =\int_{C Y_{3}} V^{A} \wedge V^{B} \wedge J, & \mathcal{K}_{A B C} & =\int_{C Y_{3}} V^{A} \wedge V^{B} \wedge V^{C} .
\end{aligned}
$$

\footnotetext{
${ }^{13}$ This generic situation can always be obtained by applying a symplectic transformation.
} 
We remark that $\mathcal{K}$ is proportional the volume $\mathcal{V}, \mathcal{K}=3 ! \mathcal{V}$, while $\mathcal{K}_{A B C}$ are the triple intersection numbers of homology four-cycles. The Hodge dual of a $(1,1)$ has the form [50]

$$
\star V^{B}=-J \wedge B^{B}+\frac{3}{2 \mathcal{K}} J \wedge J\left(\int_{C Y_{3}} V^{B} \wedge J \wedge J\right) .
$$

This allows us to evaluate the inner product of two $(1,1)$ forms:

$$
G_{A B}(M):=\frac{1}{2 \mathcal{V}} \int_{C Y_{3}} V^{A} \wedge \star V^{B}=-3\left(\frac{\mathcal{K}_{A B}}{\mathcal{K}}-\frac{3}{2} \frac{\mathcal{K}_{A} \mathcal{K}_{B}}{\mathcal{K}^{2}}\right)
$$

Using the expansion $J=M^{A} V^{A}$ of the Kähler form, we obtain

$$
\begin{aligned}
\mathcal{K} & =\mathcal{K}_{A B C} M^{A} M^{B} M^{C}=:(\mathcal{K} M M M), \quad \mathcal{K}_{A}=\mathcal{K}_{A B C} M^{B} M^{C}=:(\mathcal{K} M M)_{A} \\
\mathcal{K}_{A B} & =\mathcal{K}_{A B C} M^{C}=:(\mathcal{K} M)_{A B}
\end{aligned}
$$

so that

$$
G_{A B}(M)=-3\left(\frac{(\mathcal{K} M)_{A B}}{(\mathcal{K} M M M)}-\frac{3}{2} \frac{(\mathcal{K} M M)_{A}(\mathcal{K} M M)_{B}}{(\mathcal{K} M M M)^{2}}\right)
$$

This shows that the metric $G_{A B}(M)$ is a conical affine special real metric with Hesse potential $\mathcal{K}$, which implies that its pullbacks to hypersurfaces $\mathcal{K}=$ const. are projective special real metrics. These are the general target spaces of five-dimensional vector multiplets, which reflects that spaces of Kähler moduli with overall volumes fixed appear as the vector multiplet moduli spaces of eleven-dimensional supergravity compactified to five dimensions on a CY3 [51]. We refer to [22] for more details on special real geometry.

In type-II CY3 compactifications the Kähler moduli space, which naturally is a real space with a Hessian metric, is extended to a special Kähler or a special para-Kähler space once the $B$-field moduli are taken into account. In theories of closed oriented strings, the metric $G_{M N}$ is accompanied by the Kalb-Ramond field $B_{M N}$. For CY3 compactifications the $B$-field contributes $h^{1,1}$ real scalars associated to harmonic (1,1)-forms, which combine with the $h^{1,1}$ Kähler moduli $M^{A}$. If the $B$-field has a standard kinetic term, as is the case for type-IIA/IIA*/IIB/IIB* in signature $(1,9)$, then including the $B$-field leads to a complexification of the Kähler form and of the Kähler moduli space. The resulting moduli space is projective special Kähler, and completely determined by the underlying real Kähler moduli space by the supergravity r-map, which maps projective special real manifolds to projective special Kähler manifolds. If the sign of the kinetic term of the $B$-field is flipped, as it happens for type-IIA $(0,10)$, type-IIB' and type-IIA $(2,8)$, this induces a sign flip for the $h^{1,1} B$-field moduli. As a result, the Kähler moduli space is para-complexified rather than complexified. The resulting projective special para-Kähler manifold is obtained from the real Kähler moduli space by the para-r-map, which maps projective special real manifolds to special para-Kähler manifolds.

Thus by combining the scalar fields descending from the metric and the $B$-field, we end up either with two SK manifolds or with one SK and one SPK manifold, with all couplings encoded by two prepotentials. The remaining scalar fields extend one of these manifolds into a QK or PQK manifold. These extensions are unique, in the sense that the (P)QK manifold is obtained from an underlying $\mathrm{S}(\mathrm{P}) \mathrm{K}$ manifold by a c-map. Type-IIA and type-IIB differ 


\begin{tabular}{|l|r|r|r|}
\hline Type & $\alpha_{1}$ & $\alpha_{2}$ & $\alpha_{3}$ \\
\hline $\operatorname{IIA}_{(1,9)}$ & 1 & 1 & 1 \\
$\mathrm{IIA}_{(1,9)}^{*}$ & -1 & 1 & -1 \\
$\mathrm{IIA}_{(0,10)}$ & -1 & -1 & 1 \\
$\mathrm{IIA}_{(2,8)}$ & 1 & -1 & -1 \\
\hline
\end{tabular}

Table 14. Relative signs for kinetic terms in ten-dimensional type-IIA theories. A plus sign corresponds to a standard kinetic term in Lorentz signature, as realized in $\operatorname{IIA}_{(1,9)}$.

in that in type-IIA the vector muliplets contain the (complexified or para-complexified) Kähler moduli, while in type-IIB the vector multiplets contain the complex structure moduli. The IIA hypermultiplets contain the complex structure moduli together with the dilaton, the axion, and the R-R scalars. In type-IIA $(0,10)$ and type-IIA $(1,9)$ this leads to a QK manifold, while for type-IIA ${ }_{(1,9)}^{*}$ and for type-IIA ${ }^{(2,8)}$ a sign flip for the R-R scalars leads to a $\mathrm{PQK}_{\mathrm{SK}}$ manifold. Type-IIB hypermultiplets contain the complexified Kähler moduli for type-IIB/IIB*, which then extends to a $\mathrm{QK} / \mathrm{PQK}_{\mathrm{SK}}$ manifold, respectively. For type-IIB' the Kähler moduli space is para-complexified, and extended to a $\mathrm{PQK}_{\mathrm{SPK}}$ manifold. See table 13 for a summary.

\section{A.2 Ten-dimensional Lagrangians}

In section 3 we used the string frame parametrization of [1] to display the various type-IIA Lagrangians. ${ }^{14}$ In order to use the results of [16] and [18] on CY3 compactifications, we use the following Einstein frame parametrization:

$$
\begin{aligned}
S_{\mathrm{IIA}}=\int_{M_{10}} & \frac{1}{2} \star R_{10}-\frac{9}{16} d \log \phi \wedge \star d \log \phi-\frac{\alpha_{1}}{4} \phi^{\frac{9}{4}} d V \wedge \star d V \\
& -\frac{\alpha_{2}}{2} \phi^{-\frac{3}{2}} H_{3} \wedge \star H_{3}-\frac{\alpha_{3}}{2} \phi^{\frac{3}{4}}\left(F_{4}+d V \wedge B_{2}\right) \wedge \star\left(F_{4}+d V \wedge B_{2}\right) \\
& -\frac{\sqrt{2}}{2}\left(F_{4}+d V \wedge B_{2}\right) \wedge F_{4} \wedge B_{2}-\frac{\sqrt{2}}{6} d V \wedge B_{2} \wedge d V \wedge B_{2} \wedge B_{2} .
\end{aligned}
$$

Note that the dilaton has been redefined according to $\Phi \propto \log \phi$. Moreover we now use the same notations for form-fields as in [16] and [18]: $V$ is the RR one-form, while $F_{4}$ is the four-form field strength. The three parameters $\alpha_{i}, i=1,2,3$ encode the various sign flips, see table 14 .

As a quick check, note that this action is consistent with table 6 . We also note that the three signs are not independent, since $\alpha_{3}=\alpha_{1} \alpha_{2}$. This reflects that the three signs encode four independent theories, rather than six. For type-IIA $(0,10)$ and type-IIA $(1,9)$ it was shown in [18] that these Lagrangians arise from dimensional reduction of eleven-dimensional supergravity with signature $(1,10)$. For signature $(1,9)$ one recovers the Lagrangian of $[16]{ }^{15}$

\footnotetext{
${ }^{14}$ Complete bosonic string frame (pseudo-)Lagrangians for all type-II theories can be found in the appendix of [4].

${ }^{15}$ As remarked in [18] the second term in the third line is absent in [16], but present in [15]. It is straightforward to check that this term is generated by the field redefinition described explicitly in [18].
} 
The Lagrangians for type-IIA ${ }_{(1,9)}^{*}$ and type-IIA $(2,8)$ are obtained by a similar computation starting from the Lagrangian for eleven-dimensional supergravity in signature $(2,9)$ given in [1]. Since these Lagrangians only differ by relative signs, and since in all cases we reduce on the same manifold, we can use the results of [16] and [18] for the individual terms, and afterwards assemble them into four distinct four-dimensional Lagrangians.

\section{A.3 Reduction of the graviton-dilaton sector}

Since the Einstein-Hilbert and dilaton term are the same for all cases we can discuss them at once.

Following [16] and [18] the reduction of

$$
S_{\mathrm{EH}+\phi}=\int_{M_{10}} \frac{1}{2} \star R_{10}-\frac{9}{16} d \log \phi \wedge \star \log \phi
$$

results in

$$
S_{\mathrm{EH}+\phi}=\int_{M_{4}} \frac{1}{2} \star R_{4}-\frac{1}{2} G_{A B}(v) d v^{A} \wedge \star d v^{B}-\frac{1}{4} d \varphi \wedge \star d \varphi-g_{\alpha \bar{\beta}}(z, \bar{z}) d z^{\alpha} \wedge \star d \bar{z}^{\bar{\beta}} .
$$

Here $z^{\alpha}$ are the complex structure moduli with their special Kähler metric $g_{\alpha \beta}$ and $v^{A}$ are the real Kähler moduli with their special real metric $G_{A B}(v)$, related to the previously introduced $M^{A}$ by the field redefinition

$$
M^{A}=\sqrt{2} \phi^{-3 / 4} v^{A} .
$$

The four-dimensional dilaton $\varphi$ is related to the ten-dimensional dilaton $\phi$ by

$$
\varphi=\log \left(2 \mathcal{V} \phi^{-3}\right)
$$

Further details are given in [18].

\section{A.4 Contribution of the $B$-field to the vector multiplet sector}

Next we turn to terms descending from the $B$-field kinetic term, which arise from taking the internal part of the $B$-field to be a harmonic two-form. Following [16] and [18] we say that terms which arise from harmonic two-forms belong to the $H^{2}$-cohomology sector. These are precisely the terms which contribute to the gravity plus vector multiplet sector of the four-dimensional theory. The ten-dimensional term takes the form

$$
S_{H^{2}\left(B_{2}\right)}=\alpha_{2} \int_{M_{10}}-\left.\frac{1}{2} \phi^{-3 / 2} H_{3} \wedge \star H_{3}\right|_{H^{2}}
$$

where the sign depends on which theory we start with. We denote the projection of terms onto the $H^{2}$-cohomology sector by $\left.\right|_{H^{2}}$. Decomposing $\left.B_{2}\right|_{H^{2}}=a^{A} V^{A}$ and integrating over the CY3, we obtain

$$
S_{H^{2}\left(B_{2}\right)}=\alpha_{2} \int_{M_{4}}-\frac{1}{2} G_{A B}(v) d a^{A} \wedge \star d a^{B},
$$


where $a^{A}$ are four-dimensional scalar fields. We can combine this term with one of the terms obtained from the reduction of the Einstein-Hilbert term to obtain

$$
\int_{M_{4}}-\frac{1}{2} G_{A B}(v)\left(d v^{A} \wedge \star d v^{B}+\alpha_{2} d a^{A} \wedge \star d a^{B}\right) .
$$

Making the field redefinition

$$
v^{A}=\frac{1}{2^{1 / 6}} y^{A}, \quad a^{A}=-\frac{1}{2^{1 / 6}} x^{A}, \quad \mathcal{K}_{A B C}=c_{A B C},
$$

we can rewrite this contribution as

$$
\int_{M_{4}}-\bar{g}_{A B}(y)\left(d x^{A} \wedge \star d x^{B}+\alpha_{2} d y^{A} \wedge \star d y^{B}\right)
$$

where we have defined the new coupling matrix by

$$
\bar{g}_{A B}:=\frac{1}{2} \alpha_{2} G_{A B}=-\frac{3}{2} \alpha_{2}\left(\frac{(\text { cy })_{A B}}{(\text { cyyy })}-\frac{3}{2} \frac{(\text { cyy })_{A}(\text { cyy })_{B}}{(\text { cyyy })^{2}}\right) .
$$

\section{A.5 Contribution of R-R-kinetic terms to the vector multiplet sector}

Next we consider the contribution of the kinetic term of the R-R three-form, including its Chern-Simons like improvement term, to the $H^{2}$-sector, that is to the four-dimensional vector multiplets. Depending on which theory we start with, the ten-dimensional term is

$$
S_{H^{2}}\left(A_{3}\right)=\left.\alpha_{3} \int_{M_{10}} \frac{1}{2} \phi^{3 / 4}\left(F_{4}+d V \wedge B_{2}\right) \wedge \star\left(F_{4}+d V \wedge B_{2}\right)\right|_{H^{2}} .
$$

Following [18] we decompose $F_{4}$ and $B_{2}$ as

$$
\left.F_{4}\right|_{H^{2}}=\mathcal{F}^{A} \wedge V^{A},\left.\quad B_{2}\right|_{H^{2}}=a^{A} V^{A},
$$

where $\mathcal{F}^{A}$ are four-dimensional field strengths, $\mathcal{F}^{A}=d \mathcal{A}^{A}$. Inserting this into the above action, we obtain an action ready to integrate over:

$$
S_{H^{2}}\left(A_{3}\right)=\alpha_{3} \int_{M_{4}} \frac{1}{2} \phi^{3 / 4}\left(\mathcal{F}^{A}+a^{A} d V\right) \wedge \star\left(\mathcal{F}^{B}+a^{B} d V\right) \int_{C Y_{3}} V^{A} \wedge \star V^{B} .
$$

Performing the integral we obtain

$$
S_{H^{2}}\left(A_{3}\right)=\left.\alpha_{3} \int_{M_{4}} \frac{\sqrt{2}}{3 !} \mathcal{K}(v) G_{A B}(v)\left(\mathcal{F}^{A}+a^{A} d V\right) \wedge \star\left(\mathcal{F}^{B}+a^{B} d V\right)\right|_{H^{2}} .
$$

Similarly, the reduction of the kinetic term of the R-R one-form

$$
S_{V}=\alpha_{1} \int_{M_{10}} \frac{1}{4} \phi^{\frac{9}{4}} d V \wedge \star d V
$$

becomes

$$
S_{V}=\alpha_{1} \int_{M_{4}} \frac{\sqrt{2}}{2 \cdot 3 !} \mathcal{K}(v) \mathcal{F}^{0} \wedge \star \mathcal{F}^{0}
$$


after integration over the CY3, where we have set $\mathcal{F}^{0}=d V$ and used the field redefinition (A.7).

Using that $\alpha_{3}=\alpha_{1} \alpha_{2}$, we can combine terms as

$$
\begin{aligned}
S_{H^{2}}\left(A_{3}\right)+S_{V}= & \alpha_{1} \int_{M_{4}} \sqrt{2}\left(\frac{1}{12}(\mathcal{K} v v v)+\frac{\alpha_{2}}{6}(\mathcal{K} v v v) G_{A B} a^{A} a^{B}\right) \mathcal{F}^{0} \wedge \star \mathcal{F}^{0} \\
& +\frac{\sqrt{2} \alpha_{2}}{3}(\mathcal{K} v v v) G_{A B} a^{B} \mathcal{F}^{A} \wedge \star \mathcal{F}^{0}+\frac{\sqrt{2} \alpha_{2}}{6}(\mathcal{K} v v v) G_{A B} \mathcal{F}^{A} \wedge \star \mathcal{F}^{B},
\end{aligned}
$$

where $(\mathcal{K} v v v):=\mathcal{K}_{A B C} v^{A} v^{B} v^{C}$. Rescaling the gauge fields

$$
\mathcal{F}^{A}=\frac{1}{2^{1 / 6}} F^{A}
$$

as well as the scalars, and using (A.9), we can express the above as

$$
\begin{aligned}
S_{H^{2}}\left(A_{3}\right)+S_{V}= & \alpha_{1} \int_{M_{4}} \frac{1}{2}(\text { cyyy })\left(\frac{1}{6}+\frac{2}{3}(\text { gx } x)\right) F^{0} \wedge \star F^{0} \\
& -\frac{2}{3}(\text { cyyy })(\text { gx })_{A} F^{A} \wedge \star F^{0}+\frac{1}{3}(\text { cyyy }) g_{A B} F^{A} \wedge \star F^{B} .
\end{aligned}
$$

\section{A.6 Contribution of the topological terms to the vector multiplet sector}

The final contribution to the gravity plus vector multiplet sector comes from the topological terms

$$
S_{H^{2}(\mathrm{top})}=\int_{M_{10}}-\frac{\sqrt{2}}{2}\left(F_{4}+d V \wedge B_{2}\right) \wedge F_{4} \wedge B_{2}-\left.\frac{\sqrt{2}}{6} d V \wedge B_{2} \wedge d V \wedge B_{2} \wedge B_{2}\right|_{H^{2}} .
$$

According to [18], after reduction and field redefinitions this takes the form

$$
S_{H^{2}(\text { top })}=\int_{M_{4}} \frac{1}{6}\left[3(c x)_{A B} \mathcal{F}^{A} \wedge \mathcal{F}^{B}-3(c x x)_{A} \mathcal{F}^{A} \wedge \mathcal{F}^{0}+(c x x x) \mathcal{F}^{0} \wedge \mathcal{F}^{0}\right] .
$$

\section{A.7 Final result for the gravity and vector multiplet sector}

Combining everything obtained so far gives us the bosonic Lagrangian for the gravity multiplet and the vector multiplets:

$$
\begin{aligned}
S_{G+\mathrm{VM}}= & \int_{M_{4}} \frac{1}{2} \star R_{4}-\bar{g}_{A B}(y)\left(d x^{A} \wedge \star d x^{B}+\alpha_{2} d y^{A} \wedge \star d y^{B}\right) \\
& -\alpha_{1}\left[\frac{1}{2}(\text { cyyy })\left(\frac{1}{6}+\frac{2}{3}(\text { gxx })\right) \mathcal{F}^{0} \wedge \star \mathcal{F}^{0}\right. \\
& \left.-\frac{2}{3}(\text { cyyy })(g x)_{A} \mathcal{F}^{A} \wedge \star \mathcal{F}^{0}+\frac{1}{3}(\text { cyyy }) g_{A B} \mathcal{F}^{A} \wedge \star \mathcal{F}^{B}\right] \\
& +\frac{1}{6}\left[3(c x)_{A B} \mathcal{F}^{A} \wedge \mathcal{F}^{B}-3(c x x)_{A} \mathcal{F}^{A} \wedge \mathcal{F}^{0}+(c x x x) \mathcal{F}^{0} \wedge \mathcal{F}^{0}\right] .
\end{aligned}
$$

As shown in [25], one can introduce complex fields $z^{A}=x^{A}+i y^{A}$ if $\alpha_{2}=1$ and para-complex fields $z^{A}=x^{A}+e y^{A}$ if $\alpha_{2}=-1$. Then the second term in the first line becomes a sigma model

$$
\int_{M_{4}}-\bar{g}_{A \bar{B}}(z, \bar{z}) d z^{A} \wedge \star d \bar{z}^{\bar{B}}
$$


with a target space which is projective special Kähler for $\alpha_{2}=1$ and projective special para-Kähler for $\alpha_{2}=-1$. More generally, the results of [25] imply that the full Lagrangian can be rewritten into the form

$$
S_{G+\mathrm{VM}}=\int_{M_{4}} \frac{1}{2} \star R_{4}-\bar{g}_{A \bar{B}}(z, \bar{z}) d z^{A} \wedge \star d \bar{z}^{\bar{B}}+\frac{\alpha_{1}}{4} \mathcal{I}_{\Sigma \Lambda} F^{\Sigma} \wedge \star F^{\Lambda}+\frac{1}{4} \mathcal{R}_{\Sigma \Lambda} F^{\Sigma} \wedge F^{\Lambda},
$$

where $\Sigma, \Lambda=0,1, \ldots, n_{V}=h_{1,1}$, and where the vector coupling matrices $\mathcal{I}_{\Lambda \Sigma}$ and $\mathcal{R}_{\Lambda \Sigma}$ can be expressed by a holomorphic or para-holomorphic prepotential through the standard formulae of special geometry. This completes the derivation of the bosonic gravity plus vector multiplet Lagrangians for the four type-IIA theories. Our result indeed matches (4.1) and (4.2). For signature (1,3), where $\alpha_{2}=1$ and $\alpha_{1}=\alpha_{3}=-\lambda= \pm 1$ we obtain a complex, (projective) SK scalar manifold, and the sign of the Maxwell term distinguishes between type-IIA and type-IIA*. For signatures $(0,4)$ and $(2,2)$, where $\alpha_{2}=-1$, we obtain a para-complex, (projective) SPK manifold, and both signatures differ by the sign of the Maxwell term, which is controlled by $\alpha_{1}=-\alpha_{3}=-\lambda$. However, in these signatures the supersymmetry algebra is unique and the sign can be changed by a field redefinition [27].

\section{A.8 Contribution of the kinetic R-R-terms to the hypermultiplet sector}

We now turn to contributions from terms where the internal part is a harmonic 3-form. So far we have discussed one such term, which arises from the reduction of the Einstein-Hilbert term. This results in the sigma model $-\tilde{G}_{\alpha \bar{\beta}}(z, \bar{z}) d z^{\alpha} \wedge \star d \bar{z}^{\bar{\beta}}$, where $z^{\alpha}$ parametrize the deformations of the complex structure of the CY3, with (projective) SK metric $g_{\alpha \bar{\beta}}(z, \bar{z})$. The R-R one-form does not contribute, but there are contributions for the R-R three-form and from the Kalb-Ramond field.

We start with the contribution of the kinetic term of the four-form field strength $F_{4}=d A_{3}$,

$$
S_{H^{3}}\left(A_{3}\right)=\left.\int_{M_{1} 0} \frac{-\alpha_{3}}{2} \phi^{3 / 4} F_{4} \wedge \star F_{4}\right|_{H^{3}} .
$$

It has been shown in $[16,18]$ that

$$
\left.F_{4}\right|_{H^{3}}=d \check{A}=2^{1 / 4} d \zeta^{I} \wedge \alpha_{I}+2^{1 / 4} d \tilde{\zeta}_{I} \wedge \beta^{I}=P^{I} \wedge \Phi_{I}+\bar{Q} \wedge \bar{\Omega}+\text { h.c. }
$$

where the complex one-forms $P^{I}, \bar{Q} \in \Omega^{1}\left(M_{4}\right) \otimes \mathbb{C}$ can be expressed in terms of special geometry data associated with complex structure moduli as

$$
P^{I}=i 2^{1 / 4}\left(d \tilde{\zeta}_{J}+\mathcal{N}_{J K} d \zeta^{K}\right) N^{I J}, \quad \bar{Q}=-i 2^{1 / 4} \frac{X^{I}}{(X N \bar{X})}\left(d \tilde{\zeta}_{I}+\mathcal{N}_{I J} d \zeta^{J}\right)
$$

While the four-dimensional hypermultiplet scalars $\zeta^{I}, \tilde{\zeta}_{I}$ are defined through the expansion of $d \check{A}$ in terms of $\alpha_{I}, \beta^{I}$, the expansion of $d \check{A}$ in terms of $\Phi_{I}, \Omega$ is used to carry out the integration over the CY3:

$$
S_{H^{3}}\left(A_{3}\right)=\int_{M_{4}}-\alpha_{3} \phi^{3 / 4} P^{I} \wedge \star \bar{P}^{J} \int_{C Y_{3}} \Phi_{I} \wedge \star \bar{\Phi}_{J}+\int_{M_{4}} \alpha_{3} \phi^{3 / 4} \bar{Q} \wedge \star Q \int_{C Y_{3}} \bar{\Omega} \wedge \star \Omega .
$$

Following [18] this can be evaluated and ultimately brought to the form

$$
S_{H^{3}}\left(A_{3}\right)=\int_{M_{4}} \frac{\alpha_{3}}{2} e^{-\varphi}\left[\mathcal{I}_{I J} d \zeta^{I} \wedge \star d \zeta^{J}+\mathcal{I}^{I J}\left(d \tilde{\zeta}_{I}+\mathcal{R}_{I K} d \zeta^{K}\right) \wedge \star\left(d \tilde{\zeta}_{J}+\mathcal{R}_{J K} d \zeta^{K}\right)\right]
$$

where $\varphi$ is the four-dimensional dilaton. 


\section{A.9 Contribution of topological terms and of the $B$-field to the hypermultiplet sector}

The topological contribution to the $H^{3}$-sector comes from

$$
S_{H^{3}(\mathrm{top})}=\int_{M_{10}}-\left.\frac{\sqrt{2}}{2} F_{4} \wedge F_{4} \wedge B_{2}\right|_{H^{3}}
$$

Inserting in the expansions of these fields, we obtain

$$
S_{H^{3}(\mathrm{top})}=\int_{M_{4}}-\sqrt{2} \mathcal{B}_{2} \wedge P^{I} \wedge \bar{P}^{J} \int_{C Y_{3}} \Phi_{I} \wedge \bar{\Phi}_{J}+\int_{M_{4}} \sqrt{2} \mathcal{B}_{2} \wedge \bar{Q} \wedge Q \int_{C Y_{3}} \bar{\Omega} \wedge \Omega .
$$

Following [18] this can be evaluated and ultimately be brought to the form

$$
S_{H^{3}(\text { top })}=-\int_{M_{4}} 2 \mathcal{B}_{2} \wedge d \zeta^{I} \wedge d \tilde{\zeta}_{I}=\int_{M_{4}} 2 \mathcal{H}_{3} \wedge \zeta^{I} d \tilde{\zeta}_{I},
$$

where $\mathcal{H}_{3}=d \mathcal{B}_{2}$ is the field strength of the four-dimensional Kalb-Ramond field $\mathcal{B}_{2}$.

To this we add the contribution from the reduction of the kinetic term of the tendimensional Kalb-Ramond field. (Here the internal part is a zero-form, so this belongs to the ' $H^{0}$ sector.')

$$
S_{H^{3}(\mathrm{top})}+S_{H^{0}\left(B_{2}\right)}=\int_{M_{4}} 2 \mathcal{H}_{3} \wedge \zeta^{I} d \tilde{\zeta}_{I}-\alpha_{2} e^{2 \varphi} \mathcal{H}_{3} \wedge \star \mathcal{H}_{3} .
$$

Following [18] we dualize the four-dimensional Kalb-Ramond field $\mathcal{B}_{2}$ into a scalar field $\tilde{\phi}$.

$$
S_{H^{3}(\mathrm{top})}+S_{H^{0}(\tilde{\phi})}=-\int_{M_{4}} e^{-2 \varphi}\left[d \tilde{\phi}+\frac{1}{2}\left(\zeta^{I} d \tilde{\zeta}_{I}-\tilde{\zeta} d \zeta^{I}\right)\right] \wedge \star\left[d \tilde{\phi}+\frac{1}{2}\left(\zeta^{I} d \tilde{\zeta}_{I}-\tilde{\zeta} d \zeta^{I}\right)\right] .
$$

Note that $\alpha_{2}$ has cancelled, because in signatures $(0,10)$ and $(2,8)$, where $\alpha_{2}=-1$, the Hodge dualization generates an additional sign compared to signature $(1,9)$, where $\alpha_{2}=1$.

\section{A.10 Final result for the hypermultiplet sector}

By collecting all terms contributing to the hypermultiplet sector we obtain

$$
\begin{aligned}
S_{H}=\int_{M_{4}} & -\tilde{G}_{\alpha \bar{\beta}} d z^{\alpha} \wedge \star d \bar{z}^{\bar{\beta}}-\frac{1}{4} d \varphi \wedge \star d \varphi \\
& -e^{-2 \varphi}\left[d \tilde{\phi}+\frac{1}{2}\left(\zeta^{I} d \tilde{\zeta}_{I}-\tilde{\zeta}_{I} d \zeta^{I}\right)\right] \wedge \star\left[d \tilde{\phi}+\frac{1}{2}\left(\zeta^{I} d \tilde{\zeta}_{I}-\tilde{\zeta}_{I} d \zeta^{I}\right)\right] \\
& +\frac{\alpha_{3}}{2} e^{-\varphi}\left[\mathcal{I}_{I J} d \zeta^{I} \wedge \star d \zeta^{J}+\mathcal{I}^{I J}\left(d \tilde{\zeta}_{I}+\mathcal{R}_{I K} d \zeta^{K}\right) \wedge \star\left(d \tilde{\zeta}_{I}+\mathcal{R}_{I K} d \zeta^{K}\right)\right]
\end{aligned}
$$

where $\alpha, \beta=1, \ldots, h_{2,1}=n_{V}-1$ and $I, J=1, \ldots, n_{V}=h_{2,1}+1$. This indeed agrees with (4.3) upon identifying $\alpha_{3}=-\lambda$. This completes the derivation of the four type-IIA hypermultiplet Lagrangians from dimensional reduction. For type-IIA $(1,9)$ and type-IIA $(0,10)$, where $\alpha_{3}=-\lambda=1$, the geometry is $\mathrm{QK}\left(\alpha_{3}=1\right)$, while for type-IIA* $(1,9)$ and type$\operatorname{IIA}(2,8)$ where $\alpha_{3}=-\lambda=-1$, the geometry is PQK. In both cases the distinguished submanifold is the SK manifold provided by the complex structure moduli space. 
Open Access. This article is distributed under the terms of the Creative Commons Attribution License (CC-BY 4.0), which permits any use, distribution and reproduction in any medium, provided the original author(s) and source are credited.

\section{References}

[1] C.M. Hull, Timelike $T$ duality, de Sitter space, large $N$ gauge theories and topological field theory, JHEP 07 (1998) 021 [hep-th/9806146] [INSPIRE].

[2] C.M. Hull, Duality and the signature of space-time, JHEP 11 (1998) 017 [hep-th/9807127] [INSPIRE].

[3] C.M. Hull and R.R. Khuri, Branes, times and dualities, Nucl. Phys. B 536 (1998) 219 [hep-th/9808069] [INSPIRE].

[4] R. Dijkgraaf, B. Heidenreich, P. Jefferson and C. Vafa, Negative Branes, Supergroups and the Signature of Spacetime, JHEP 02 (2018) 050 [arXiv: 1603.05665] [INSPIRE].

[5] R. Blumenhagen, M. Brinkmann, A. Makridou, L. Schlechter and M. Traube, dS Spaces and Brane Worlds in Exotic String Theories, JHEP 06 (2020) 077 [arXiv:2002.11746] [InSPIRE].

[6] B. de Wit and H. Nicolai, Hidden Symmetry in d=11 Supergravity, Phys. Lett. B 155 (1985) 47 [INSPIRE].

[7] G.W. Moore, Finite in all directions, hep-th/9305139 [INSPIRE].

[8] O. Hohm, S.K. Kwak and B. Zwiebach, Double Field Theory of Type II Strings, JHEP 09 (2011) 013 [arXiv: 1107.0008] [INSPIRE].

[9] O. Hohm and H. Samtleben, The many facets of exceptional field theory, PoS CORFU2018 (2019) 098 [arXiv: 1905.08312] [InSPIRE].

[10] L. Gall and T. Mohaupt, Supersymmetry algebras in arbitrary signature and their R-symmetry groups, JHEP 10 (2021) 203 [arXiv:2108.05109] [INSPIRE].

[11] M. Kontsevich and G. Segal, Wick Rotation and the Positivity of Energy in Quantum Field Theory, Quart. J. Math. Oxford Ser. 72 (2021) 673 [arXiv:2105.10161] [INSPIRE].

[12] E. Witten, A Note On Complex Spacetime Metrics, arXiv:2111.06514 [InSPIRE].

[13] J.-L. Lehners, Allowable complex metrics in minisuperspace quantum cosmology, Phys. Rev. D 105 (2022) 026022 [arXiv:2111.07816] [INSPIRE].

[14] T. Mohaupt and K. Waite, Euclidean Actions, Instantons, Solitons and Supersymmetry, J. Phys. A 44 (2011) 175403 [arXiv: 1011.6301] [InSPIRE].

[15] S. Ferrara and S. Sabharwal, Dimensional Reduction of Type II Superstrings, Class. Quant. Grav. 6 (1989) L77 [INSPIRE].

[16] M. Bodner, A.C. Cadavid and S. Ferrara, $(2,2)$ vacuum configurations for type IIA superstrings: $N=2$ supergravity Lagrangians and algebraic geometry, Class. Quant. Grav. 8 (1991) 789 [INSPIRE].

[17] R. Bohm, H. Gunther, C. Herrmann and J. Louis, Compactification of type IIB string theory on Calabi-Yau threefolds, Nucl. Phys. B 569 (2000) 229 [hep-th/9908007] [INSPIRE].

[18] W. Sabra and O. Vaughan, 10D to 4D Euclidean Supergravity over a Calabi-Yau three-fold, Class. Quant. Grav. 33 (2016) 015010 [arXiv:1503.05095] [INSPIRE]. 
[19] W.A. Sabra, Special geometry and space-time signature, Phys. Lett. B 773 (2017) 191 [arXiv: 1706.05162] [INSPIRE].

[20] L. Andrianopoli et al., $N=2$ supergravity and $N=2$ superYang-Mills theory on general scalar manifolds: Symplectic covariance, gaugings and the momentum map, J. Geom. Phys. 23 (1997) 111 [hep-th/9605032] [INSPIRE].

[21] D.Z. Freedman and A. Van Proeyen, Supergravity, Cambridge University Press (2012).

[22] G.L. Cardoso and T. Mohaupt, Special geometry, Hessian structures and applications, Phys. Rept. 855 (2020) 1 [arXiv: 1909.06240] [INSPIRE].

[23] E. Lauria and A. Van Proeyen, $\mathcal{N}=2$ Supergravity in $D=4,5,6$ Dimensions, Lect. Notes Phys. 966 (2020) 1 [arXiv: 2004.11433] [InSPIRE].

[24] V. Cortes, C. Mayer, T. Mohaupt and F. Saueressig, Special geometry of Euclidean supersymmetry. 1. Vector multiplets, JHEP 03 (2004) 028 [hep-th/0312001] [INSPIRE].

[25] V. Cortes and T. Mohaupt, Special Geometry of Euclidean Supersymmetry III: The Local r-map, instantons and black holes, JHEP 07 (2009) 066 [arXiv: 0905.2844] [INSPIRE].

[26] V. Cortés, P. Dempster, T. Mohaupt and O. Vaughan, Special Geometry of Euclidean Supersymmetry IV: the local c-map, JHEP 10 (2015) 066 [arXiv: 1507.04620] [INSPIRE].

[27] V. Cortés, L. Gall and T. Mohaupt, Four-dimensional vector multiplets in arbitrary signature (I), Int. J. Geom. Meth. Mod. Phys. 17 (2020) 2050150 [arXiv:1907.12067] [inSPIRE].

[28] S. Cecotti, S. Ferrara and L. Girardello, Geometry of Type II Superstrings and the Moduli of Superconformal Field Theories, Int. J. Mod. Phys. A 4 (1989) 2475 [inSPIRE].

[29] S. Ferrara and S. Sabharwal, Quaternionic Manifolds for Type II Superstring Vacua of Calabi-Yau Spaces, Nucl. Phys. B 332 (1990) 317 [InSPIRE].

[30] J. Gutowski, T. Mohaupt and G. Pope, From static to cosmological solutions of $\mathcal{N}=2$ supergravity, JHEP 08 (2019) 172 [arXiv:1905.09167] [INSPIRE].

[31] J. Gutowski, T. Mohaupt and G. Pope, Cosmological Solutions, a New Wick-Rotation, and the First Law of Thermodynamics, JHEP 03 (2021) 293 [arXiv: 2008.06929] [INSPIRE].

[32] D. Klemm and M. Nozawa, Geometry of Killing spinors in neutral signature, Class. Quant. Grav. 32 (2015) 185012 [arXiv: 1504.02710] [INSPIRE].

[33] W.A. Sabra, Phantom Metrics With Killing Spinors, Phys. Lett. B 750 (2015) 237 [arXiv: 1507.04597] [INSPIRE].

[34] J.B. Gutowski and W.A. Sabra, Real Killing Spinors in Neutral Signature, JHEP 11 (2019) 173 [arXiv: 1905.01910] [INSPIRE].

[35] W.A. Sabra, Kasner Branes with Arbitrary Signature, Phys. Lett. B 809 (2020) 135694 [arXiv: 2005.03953] [INSPIRE].

[36] W.A. Sabra, Hypersymplectic geometry and supersymmetric solutions in $(t, s) 5 D$ supergravity, Phys. Rev. D 104 (2021) 046012 [arXiv:2105.00806] [InSPIRE].

[37] W.A. Sabra, Flow Equations In Arbitrary Signature, arXiv:2111.05141 [INSPIRE].

[38] E. Witten, The Search for Higher Symmetry in String Theory, Phil. Trans. Roy. Soc. Lond. A 329 (1989) 349 [INSPIRE].

[39] B. de Wit and A. Van Proeyen, Potentials and Symmetries of General Gauged $N=2$ Supergravity: Yang-Mills Models, Nucl. Phys. B 245 (1984) 89 [INSPIRE]. 
[40] B. de Wit, P.G. Lauwers and A. Van Proeyen, Lagrangians of $N=2$ Supergravity-Matter Systems, Nucl. Phys. B 255 (1985) 569 [inSPIRE].

[41] V. Cortes, C. Mayer, T. Mohaupt and F. Saueressig, Special geometry of euclidean supersymmetry. II. Hypermultiplets and the c-map, JHEP 06 (2005) 025 [hep-th/0503094] [INSPIRE].

[42] M.A. Lledó, O. Macia, A. Van Proeyen and V.S. Varadarajan, Special geometry for arbitrary signatures, IRMA Lect. Math. Theor. Phys. 16 (2010) 85 [hep-th/0612210] [INSPIRE].

[43] T. Ortin, Gravity and Strings, Cambridge University Press (2004) [DOI].

[44] B.R. Greene, String theory on Calabi-Yau manifolds, in Theoretical Advanced Study Institute in Elementary Particle Physics (TASI 96): Fields, Strings, and Duality, pp. 543-726 (1996) [hep-th/9702155] [INSPIRE].

[45] J. Dai, R.G. Leigh and J. Polchinski, New Connections Between String Theories, Mod. Phys. Lett. A 4 (1989) 2073 [INSPIRE].

[46] P. Hořava, Background Duality of Open String Models, Phys. Lett. B 231 (1989) 251 [InSPIRE].

[47] J. Louko and R.D. Sorkin, Complex actions in two-dimensional topology change, Class. Quant. Grav. 14 (1997) 179 [gr-qc/9511023] [INSPIRE].

[48] S. Bondarenko, Dynamical signature: complex manifolds, gauge fields and non-flat tangent space, arXiv:2111.06095 [INSPIRE].

[49] T. Hubsch, Calabi-Yau Manifolds, World Scientific (1991).

[50] P. Candelas and X. de la Ossa, Moduli Space of Calabi-Yau Manifolds, Nucl. Phys. B 355 (1991) 455 [INSPIRE].

[51] A.C. Cadavid, A. Ceresole, R. D'Auria and S. Ferrara, Eleven-dimensional supergravity compactified on Calabi-Yau threefolds, Phys. Lett. B 357 (1995) 76 [hep-th/9506144] [INSPIRE]. 